\title{
Disentangling Self-Atomic Motions in Polyisobutylene by Molecular Dynamics Simulations
}

\author{
Yasmin Khairy ${ }^{1,2}\left(\mathbb{D}\right.$, Fernando Alvarez ${ }^{2,3,4}(\mathbb{D})$, Arantxa Arbe ${ }^{2,3}(\mathbb{D})$ and Juan Colmenero $2,3,4,5, *(\mathbb{D})$ \\ 1 Physics Department, Faculty of Science, Zagazig University, Zagazig 44519, Egypt; yasmin_ph@yahoo.com \\ 2 Centro de Física de Materiales (CSIC, UPV /EHU), Paseo Manuel de Lardizabal 5, E-20018 San Sebastián, \\ Spain; fernando.alvarez@ehu.eus (F.A.); a.arbe@ehu.eus (A.A.) \\ 3 Materials Physics Center MPC, Paseo Manuel de Lardizabal 5, E-20018 San Sebastián, Spain \\ 4 Departamento de Polímeros y Materiales Avanzados: Física, Química y Tecnología (UPV/EHU), \\ Apartado 1072, E-20080 San Sebastián, Spain \\ 5 Donostia International Physics Center (DIPC), Paseo Manuel de Lardizabal 4, E-20018 San Sebastián, Spain \\ * Correspondence: juan.colmenero@ehu.eus
}

check for

updates

Citation: Khairy, Y.; Alvarez, F.;

Arbe, A.; Colmenero, J. Disentangling Self-Atomic Motions in

Polyisobutylene by Molecular Dynamics Simulations. Polymers 2021, 13, 670. https://doi.org/10.3390/ polym 13040670

Academic Editor: Roland G. Winkler Received: 19 January 2021 Accepted: 17 February 2021 Published: 23 February 2021

Publisher's Note: MDPI stays neutral with regard to jurisdictional clai$\mathrm{ms}$ in published maps and institutional affiliations.

Copyright: () 2021 by the authors. Licensee MDPI, Basel, Switzerland. This article is an open access article distributed under the terms and conditions of the Creative Commons Attribution (CC BY) license (https:// creativecommons.org/licenses/by/ $4.0 /)$.

\begin{abstract}
We present fully atomistic molecular dynamics simulations on polyisobutylene (PIB) in a wide temperature range above the glass transition. The cell is validated by direct comparison of magnitudes computed from the simulation and measured by neutron scattering on protonated samples reported in previous works. Once the reliability of the simulation is assured, we exploit the information in the atomic trajectories to characterize the dynamics of the different kinds of atoms in PIB. All of them, including main-chain carbons, show a crossover from Gaussian to non-Gaussian behavior in the intermediate scattering function that can be described in terms of the anomalous jump diffusion model. The full characterization of the methyl-group hydrogen motions requires accounting for rotational motions. We show that the usually assumed statistically independence of rotational and segmental motions fails in this case. We apply the rotational rate distribution model to correlation functions calculated for the relative positions of methyl-group hydrogens with respect to the carbon atom at which they are linked. The contributions to the vibrational density of states are also discussed. We conclude that methyl-group rotations are coupled with the main-chain dynamics. Finally, we revise in the light of the simulations the hypothesis and conclusions made in previously reported neutron scattering investigations on protonated samples trying to address the origin of the dielectric $\beta$-process.
\end{abstract}

Keywords: dynamics of polymers; fully atomistic molecular dynamics simulations

\section{Introduction}

During the last decades, neutron scattering (NS) has proved to be an extremely valuable microscopic technique to decipher the structural and dynamical properties of glass-forming systems, including polymeric materials [1-3]. In particular, its sensitivity to hydrogen motions allows characterizing the molecular dynamics of protonated systems at length scales in the $\AA$ to $\mathrm{nm}$ range. At such scales, well above the glass-transition temperature $T_{g}$ the structural or $\alpha$-relaxation is the most relevant dynamical process. In the early 90's, by using backscattering techniques on protonated samples [poly(vinyl methyl ether) (PVME), phenoxy (PH) and poly(vinyl chloride) (PVC)], the correlation function corresponding to self-atomic (hydrogen) motions in this regime was characterized by stretched exponentials with similar stretching exponents ( $\beta$-parameter) as those obtained by dielectric relaxation for the $\alpha$-process [4]. The momentum transfer $(Q)$ dependence of the characteristic times was found to be correlated with the shape parameter $\beta$ such that the atomic probability distributions could be approximately described by Gaussian functions. The underlying motions were qualified as anomalous diffusion-like processes where the atomic mean squared displacement $\left\langle r^{2}\right\rangle$ increases sublinearly with 
time $\left(\left\langle r^{2}\right\rangle \propto t^{\beta}\right)$. These results were later confirmed by similar experiments on 'canonical' polymers [like e.g., 1,4-polybutadiene (PB), poly(vinyl ethylene) (PVE), polyisoprene (PI) and polyisobutylene (PIB)] [5], and also on other systems like ionic liquids [6,7], alcohols [8], nanocomposites [9,10], hydration water [11] or other complex macromolecules like lignin [12]. Backscattering measurements are however restricted to a rather limited dynamic window and the results are affected by the instrumental resolution function, which hampers an accurate determination of the spectral shape. Deconvolution from the instrumental resolution is possible by the neutron spin echo (NSE) technique, which in addition accesses the widest dynamic range for NS instruments. The first NSE results on a fully protonated polymer were reported in Ref. [13]. The sample was PIB. Though still subjected to uncertainties in the determination of the exact value of the shape parameter $\beta$, the NSE data revealed unambiguously stretched functional forms and $Q$-dependences of the characteristic times compatible with the Gaussian approximation, confirming the previous general conclusions. In the high $Q$-range investigated, however, indications for deviations from Gaussian behavior were found, suggesting a crossover from Gaussian to non-Gaussian behavior in a $Q$-range of about $1 \AA^{-1}$.

Such deviations were investigated in more detail on PI with deuterated methyl groups by combining NS (backscattering and NSE) experiments with molecular dynamics (MD) simulations [14,15]. This combined approach results to be an extremely powerful tool, as it has been shown in a series of systems during the last years (see, e.g., $[3,16])$. To explain the behavior found for the hydrogen motions in PI, the so-called anomalous jump diffusion model was proposed. This model is a generalization of the well-known jump-diffusion model [17] to the case of sublinear diffusion. Similar crossover from Gaussian to non-Gaussian behavior was also later reported for other glass-forming polymers like PB [18], PVE [18,19], PVME [20], poly(methyl methacrylate) (PMMA) [21], poly(ethylene propylene) (PEP) [22], head-to-head polypropylene (hh-PP) [22], poly(vinyl acetate) (PVAc) [23], poly(vinyl pirrolidone) (PVP) [24], poly(ethyl methacrylate) (PEMA) [25] and poly(tetrahydrofurane) (PTHF) [26], as well as for other systems as e.g., n-alkanes [27] or H-bonding liquids [28-30]. The anomalous jump diffusion model has also been applied to some of these polymers and seems to be successful in capturing the essence of the caging effect occurring in these systems in a very simple way. The limited $Q$-range of the NSE experiments on PIB [13] prevented however a reliable application of such model.

In addition to the $\alpha$-relaxation, other processes like the $\beta$-relaxation might be found in polymers. In two cases, PB and PIB, there were attempts to characterize by NS the molecular motions behind the $\beta$-process. This is an extremely difficult task, since in the relatively high frequency window accessed by NS, contributions not only from the $\beta$-process but also from the $\alpha$-relaxation are expected to be present. For PB, coherent scattering data obtained on a deuterated sample were analyzed assuming a scenario of statistically independent $\alpha$ and $\beta$-processes [31,32]. The localized motions behind the $\beta$-relaxation could be characterized as rotational jumps of the chain building blocks around their center of mass. This was corroborated by MD-simulations some years later [33]. Such an approach was also applied to describe the NS results on PIB. From the description of the self-correlation function accessed on a protonated sample, the length of the jumps involved in the localized motions giving rise to the dielectric $\beta$-process was determined to be of about $2.7 \AA$ [34]. However, NSE experiments on a fully deuterated sample addressing collective relaxation in PIB and evaluated in the same framework delivered a much smaller value (0.5-0.9 $\AA$ ) for such jump distance [35]. Conversely, the dielectrically detected $\beta$ relaxation showed very similar features as the so-called $\delta$-process reported from NMR experiments [36-38], that was interpreted as rotations of methyl groups (which are not dielectrically active). Interestingly, the NS spectra of PIB in the vicinity of $T_{g}$ do not show any clear hint of classical hopping of its methyl groups [39,40], as it is usually the case in glass-forming polymers [41]. The NS experimental signature of methyl group dynamics in PIB is the presence of a pronounced, broad and structured peak in the vibrational density of states (VDOS) measured on fully protonated samples [39,42]. Such data in fact reveal 
the total-hydrogen averaged VDOS, containing contributions from both, methyl-group and main-chain hydrogens. The reported peak is located at an energy of about $40 \mathrm{meV}$, i.e., a rather high value as compared to the typical energies corresponding to the methyl-group librational peaks in polymers [41]. This observation was explained in Ref. [39] in terms of a strong coupling between the methyl groups, which also may be related to the strong steric hindrance of the polymer chain in PIB. Adams et al. [42] proposed that the peak at $\approx 40 \mathrm{meV}$ was due to a broadened methyl group torsional frequency, and the structure observed at $25 \mathrm{~K}$ (a kind of shoulder or additional peak at higher frequencies) was due mainly to the C-C-C skeletal vibrations being in the same region of the spectrum. They remarked the importance of using selective deuteration to experimentally resolve possible different components of the structured broad peak observed.

Polyisobutylene is thus one of the glass-forming polymers that has been most extensively investigated by NS [13,34,35,39,40,42-54]. At the same time, however, there are still a number of open questions regarding the interpretation of these results. In addition, we cannot forget that PIB is a very interesting polymer also for industrial applications due to its well-known and unusual physical properties; remarkably low $T_{g} \approx 200 \mathrm{~K}$ resulting in low cost processability, high packing efficiency and low gas permeability. Any contribution to the microscopic understanding of the underlying dynamical processes in this system is always important.

With these ideas in mind, we have performed fully atomistic MD-simulations on PIB in a wide temperature range above $T_{g}$. We have followed the strategy that has proved to be highly successful in previous studies that combined NS and MD-simulations $[3,16]$. First, the cell is validated by direct comparison of magnitudes computed from the simulation and measured by NS. We addressed problems related with collective features in a previous work [55]. There, the realism of the simulated cell was checked by comparison of data corresponding to the static and dynamic structure factor. Here we focus on self atomic motions. Consistently, the validation will be performed against NS results on protonated samples mainly determined by the self-correlation function of the hydrogens. Once the reliability of the simulated cell is assured, with all the information contained in the simulations at hand, we can ask questions that an experimentalist cannot answer due to technical limitations. In particular, we can address how good are the approximations involved in the experimental data analysis (always a crucial problem).

This work is focused on the characterization of the dynamics of the different kinds of atoms in PIB. In theory, partial deuteration of the sample allows selectively investigating by NS the motions of the remaining hydrogens. However, partially deuterated PIB samples are not easy to synthesize and consequently there are no available NS results that allow isolating main-chain from methyl-group hydrogen motions. Moreover, since the incoherent scattering cross-section of carbon atoms is 0 , their self-motions are inherently inaccessible by NS. One particularly interesting question is how different are the motions of the total subensamble of hydrogens from those of the main-chain atoms. Do the latter also show deviations from Gaussian behavior? Along this paper, we first discuss the dynamic heterogeneity associated to the different atomic species and the applicability of the anomalous jump diffusion model to the different kinds of atoms in PIB. Thereafter, the characterization of the methyl-group hydrogen motions is performed. We note that these motions are very commonly found to contribute the the quasielastic spectra together with other processes like the segmental relaxation (see, e.g., [56-62]) and these contributions are not easy to disentangle. We show that the usually assumed statistically independence of rotational and segmental motions is inappropriate for PIB. Exploiting the possibility offered by the simulations of calculating correlation functions corresponding to relative motions of atoms (including those defined in real space), we apply the rotational rate distribution model and discuss the particularities of PIB methyl-group dynamics. The contributions to the VDOS are also disentangled. Finally, we revise in the light of the simulations the hypotheses and conclusions made in previously reported neutron scattering investigations on protonated samples trying to address the origin of the dielectric $\beta$-process of this polymer. 


\section{Molecular Dynamics Simulations}

Fully atomistic molecular dynamics simulations were carried out using the COMPASS forcefield implemented within the commercial software package MATERIALS STUDIO 5.1. The cubic cell contained 20 PIB chains of 70 monomers $\left(M_{w}=3923 \mathrm{~g} / \mathrm{mol}\right.$, i.e., smaller than the entanglement mass $M_{e} \approx 7000 \mathrm{~g} / \mathrm{mol}$ [63], and a total number of atoms $N=16,840$ ). The system was simulated at the temperatures of 470, 390, 365, 335 and $320 \mathrm{~K}$, following the procedure explained in Ref. [55], to carry out 100 ns dynamics.

\section{Computed Magnitudes}

The trajectory in space of a single atom is described by the self part of the van Hove correlation function $G_{s}(\vec{r}, t) . G_{s}(\vec{r}, t)$ is the probability to find an atom at time $t$ at a position $\vec{r}$ if it was at $\vec{r}=0$ for $t=0$ :

$$
G_{S}(\vec{r}, t)=\frac{1}{N}\left\langle\sum_{i=1}^{N} \delta\left[\vec{r}-\left(\vec{r}_{i}(t)-\vec{r}_{i}(0)\right)\right]\right\rangle .
$$

Here $\vec{r}_{i}$ is the position of the atom $i$ and $N$ the number of nuclei in the sample. The brackets denote the ensemble average. The Fourier transform of $G_{s}(\vec{r}, t)$ in $\vec{Q}$-space is the so-called intermediate scattering function $F_{s}(\vec{Q}, t)$,

$$
F_{S}(\vec{Q}, t)=\int G_{s}(\vec{r}, t) \exp (i \vec{Q} \vec{r}) d \vec{r} .
$$

For some simple cases-free nuclei in a gas, harmonic crystals, simple diffusion at long times; $G_{S}(\vec{r}, t)$ is a Gaussian function [64,65]; in an isotropic system this implies

$$
G_{s}^{\text {gauss }}(r, t)=\left[\frac{\alpha(t)}{\pi}\right]^{3 / 2} \exp \left[-\alpha(t) r^{2}\right] .
$$

In the Gaussian approximation the even moments of $G_{s}(r, t)$

$$
\left\langle r^{2 n}\right\rangle=\int_{0}^{\infty} r^{2 n} G_{s}(r, t) 4 \pi r^{2} d r
$$

can be straightforwardly calculated. For instance, the mean squared displacement of the atom $\left\langle r^{2}(t)\right\rangle$ is given by $\left\langle r^{2}(t)\right\rangle=3 /[2 \alpha(t)]$. Moreover, in such Gaussian case $F_{s}(Q, t)$ is entirely determined by $\left\langle r^{2}(t)\right\rangle$ :

$$
F_{s}^{\text {gauss }}(Q, t)=\exp \left[-\frac{\left\langle r^{2}(t)\right\rangle}{6} Q^{2}\right] .
$$

In more general cases, deviations of $G_{s}(r, t)$ from the Gaussian form [Equation ((3)] may be expected. $F_{S}(Q, t)$ can then be expressed in terms of its expansion in $Q$ (see, e.g., Ref. [66])

$$
F_{S}(Q, t)=\exp \left[-\frac{\left\langle r^{2}(t)\right\rangle}{6} Q^{2}+\frac{\alpha_{2}(t)\left\langle r^{2}(t)\right\rangle^{2}}{72} Q^{4}+\ldots\right]
$$

where $\alpha_{2}(t)$ giving the leading correction is the so called second order non-Gaussian parameter. It is defined as [67]

$$
\alpha_{2}(t)=\frac{3}{5} \frac{\left\langle r^{4}(t)\right\rangle}{\left\langle r^{2}(t)\right\rangle^{2}}-1 .
$$

Evidently, in the Gaussian case $\alpha_{2}(t)=0$.

\section{Validation}

As we will compare MD-simulation with experimental neutron scattering (NS) results, we will introduce some magnitudes and functions related with NS that will be invoked. In general the neutron intensity scattered by a sample contains an incoherent contribution reflecting the self motion of atoms, as above defined, and a coherent contribution related to 
the atomic pair correlations. These contributions are weighted by the factors $I_{i n c}$ and $I_{\text {coh }}(Q)$ respectively. These factors depend on the incoherent and coherent scattering lengths $b_{i}^{\text {inc }}$ and $b_{i}^{c o h}$ of the nuclei in the sample ( $i$ refers to the nucleus considered) as:

$$
I_{i n c}=\frac{1}{N} \sum_{i}^{N}\left(b_{i}^{i n c}\right)^{2}
$$

and

$$
I_{c o h}(Q)=\frac{1}{N}\left\langle\sum_{i}^{N} \sum_{j}^{N} b_{i}^{c o h} b_{j}^{c o h} e^{i \vec{Q}\left(\vec{r}_{i}-\vec{r}_{j}\right)}\right\rangle
$$

Here, $\vec{r}_{i}$ and $\vec{r}_{j}$ are taken at the same time and the brackets mean the thermal average.

Hydrogen presents a very large value for the incoherent scattering length $\left(b_{H}^{i n c}=25.274 \mathrm{fm}\right)$. In a fully protonated polymer sample consisting of $\mathrm{H}$ and $\mathrm{C}$, the scattered intensity is completely dominated by the incoherent contribution from the protons ( $C$ only scatters coherently with $b_{C}^{c o h}=6.6511 \mathrm{fm}$, and $\left.b_{H}^{c o h}=-3.7406 \mathrm{fm}\right)$. Therefore, in such case $I_{i n c}>>I_{c o h}(Q)$ for all $Q$-values and NS results are predominantly determined by $F_{S}(Q, t)$ related to the self motion of the hydrogens.

In this work, to validate the simulated cell we have used previously published neutron spin echo (NSE) results on fully hydrogenated PIB [13]. NSE is distinguished from all other dynamic neutron scattering techniques in that it measures time-dependent correlation functions. The NSE signal is given by $[68,69]$ :

$$
S_{N S E}(Q, t)=\frac{I_{c o h}(Q) \tilde{F}(Q, t)-\frac{1}{3} I_{i n c} F_{S}(Q, t)}{I_{c o h}(Q)-\frac{1}{3} I_{i n c}}
$$

where $\tilde{F}(Q, t)$ and $F_{S}(Q, t)$ are the intermediate pair correlation function (normalized to its value at $t=0$ ) and the self correlation function above defined respectively.

Figure 1 shows the comparison between the experimental results measured by NSE on a fully hydrogenated sample and computed from the atomic trajectories in the simulation following Equation (10). The agreement is excellent. The simulations fairly reproduce the $Q$ - and $T$-dependence of the characteristic times and spectral shape experimentally observed. It is noteworthy that no shift in timescale has been imposed for a direct matching.

Though the main focus of this work is on the dynamical behavior at times longer than the picosecond, we will also discuss some aspects related with the vibrational processes. Therefore, we have also considered the vibrational density of states (VDOS, $Z(E)$ ) to validate the simulated cell. The VDOS of PIB was investigated applying NS to fully protonated samples (revealing the VDOS corresponding to all the hydrogens in the system) by Frick et al. [39] at $240 \mathrm{~K}$ and later by Adams et al. [42] at $25 \mathrm{~K}$, using better resolution. These data are reproduced in Figure 2 in the region below $150 \mathrm{meV}$.

From the simulations, the $Z(E)$-function can be calculated from the spectral density of the velocity autocorrelation function as

$$
Z(E) \propto \int_{-\infty}^{+\infty} e^{-i E t}\langle\vec{v}(0) \vec{v}(t)\rangle d t
$$

where the velocity autocorrelation function is calculated in terms of the velocity autocorrelation function of each (Hydrogen) atom as:

$$
\langle\vec{v}(0) \vec{v}(t)\rangle=\frac{1}{N} \sum_{i}\left\langle\vec{v}_{i}(0) \vec{v}_{i}(t)\right\rangle
$$




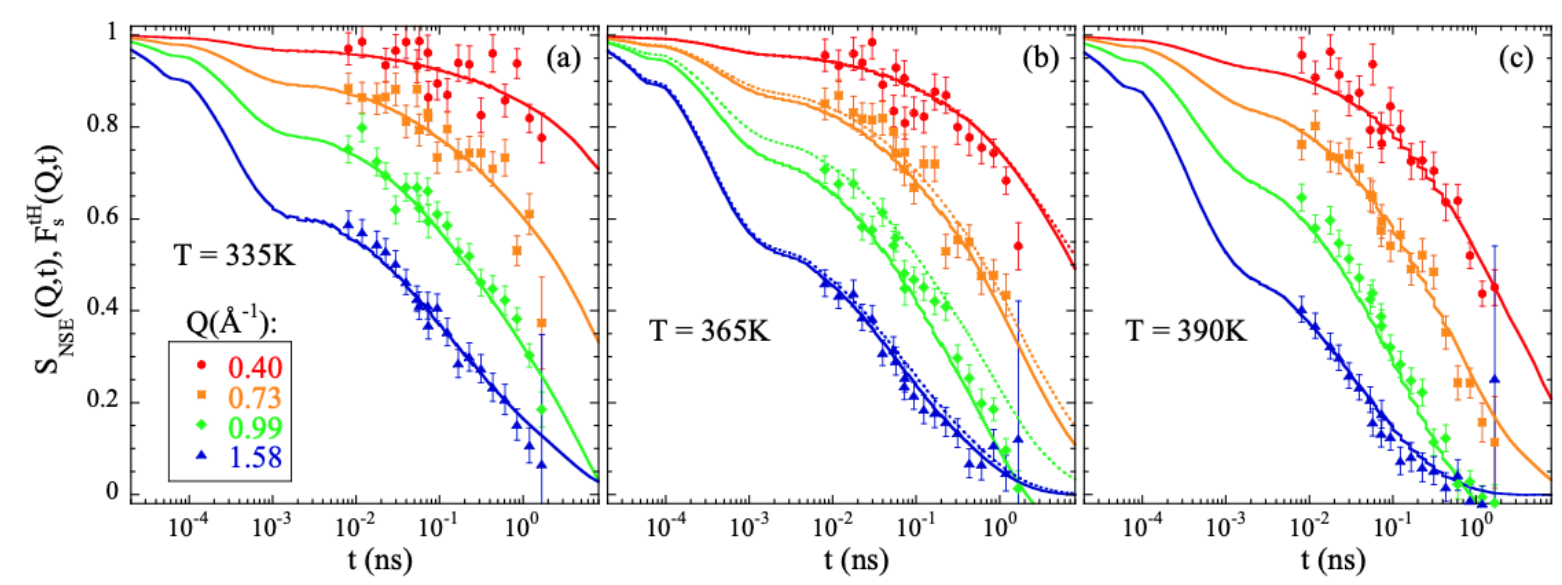

Figure 1. Comparison of the neutron spin echo results obtained from measurements on a fully hydrogenated PIB sample [13] (symbols) and calculated from the simulations (solid lines) at $335 \mathrm{~K}(\mathbf{a}), 365 \mathrm{~K}(\mathbf{b})$ and $390 \mathrm{~K}$ (c) at the Q-values indicated in (a). Bandpass corrections have been applied to the experimental data (see, e.g., [19]). The value considered for the band pass time was $0.2 \mathrm{ps}$. For comparison, the $F_{S}(Q, t)$ calculated for all hydrogens at $365 \mathrm{~K}$ is shown by the dotted lines in (b).

The $Z(E)$ was calculated running a NVT dynamics of 800 ps at $25 \mathrm{~K}$. However, we note that equilibrating a simulated cell at $25 \mathrm{~K}$ is just impossible. Thus the dynamics was run at $25 \mathrm{~K}$ but in the cell previously equilibrated at $320 \mathrm{~K}$, i.e., with the density and the structure corresponding to $320 \mathrm{~K}$. Data were recorded every $0.01 \mathrm{ps}$. The comparison with the results of Ref. [42] is presented in Figure 2. The overall agreement found is rather good, supporting the realism of the simulations also regarding vibrational aspects. We note that there are no quantum mechanic corrections in MD-simulations, which should mainly affect the high-energy region of the spectrum. Therefore, the comparison has to be taken with care in such a region.

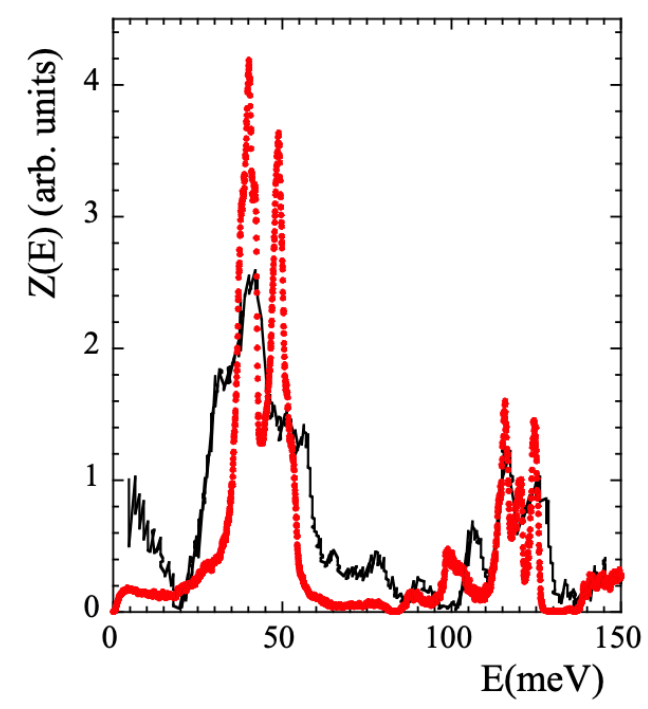

Figure 2. Comparison of the VDOS obtained from measurements on a fully hydrogenated PIB sample [42] (black line) and calculated from the simulations (red circles) at $25 \mathrm{~K}$.

\section{Results and Discussion}

Once the cell has been properly validated, from the MD-trajectories it is possible to calculate also magnitudes that are not easily (or not at all) experimentally accessible, like e.g., correlation functions in real space, or analyzing separately the different kinds of nuclei (including the 'experimentally invisible' carbons) in the sample. This information can be of utmost help to interpret experimental observations, and/or to check different theoretical frameworks. Such capabilities of the simulations are exploited in this section. 


\subsection{Distinguishing the Different Atomic Species: Chemical Dynamic Heterogeneity in PIB}

For a $Q$-value of $1.5 \AA^{-1}$ and $T=335 \mathrm{~K}$, Figure 3 a shows the intermediate incoherent scattering function of the different kinds of atoms in PIB: main-chain carbons (cC), mainchain hydrogens $(\mathrm{cH})$, methyl-group carbons $(\mathrm{mC})$ and methyl-group hydrogens $(\mathrm{mH})$ (see inset in Figure 3a for the definitions). With $\mathrm{tH}$ we denote the total hydrogens (main-chain and methyl-group hydrogens). Figure 3a reveals that: (i) for any type of atom the scattering function decays in two main steps, before and after $\approx 1$ ps; (ii) the curves completely decay to 0 in the dynamic window investigated in this temperature range. The achievement of such a full decorrelation can be attributed to the dynamics of the $\alpha$-relaxation; (iii) the atomic motions in PIB are very heterogeneous. Defining a global characteristic time $\tau_{0.2}$ as that at which the function reaches the value of 0.2 (see dotted line in the figure), at $335 \mathrm{~K}$ we find a difference of more than one order of magnitude between $\tau_{0.2}^{m H}$ and $\tau_{0.2}^{c C}$. (iv) the decay at short times is more pronounced for methyl-group atoms than for main-chain atoms, and (v) the stretching of the second decay is much stronger for $\mathrm{mH}$ than for the other atoms in the system. Finally, as expected, the behavior of $\mathrm{tH}$ is very close to that of $\mathrm{mH}-6$ out of the 8 hydrogens in PIB are located in the methyl groups.
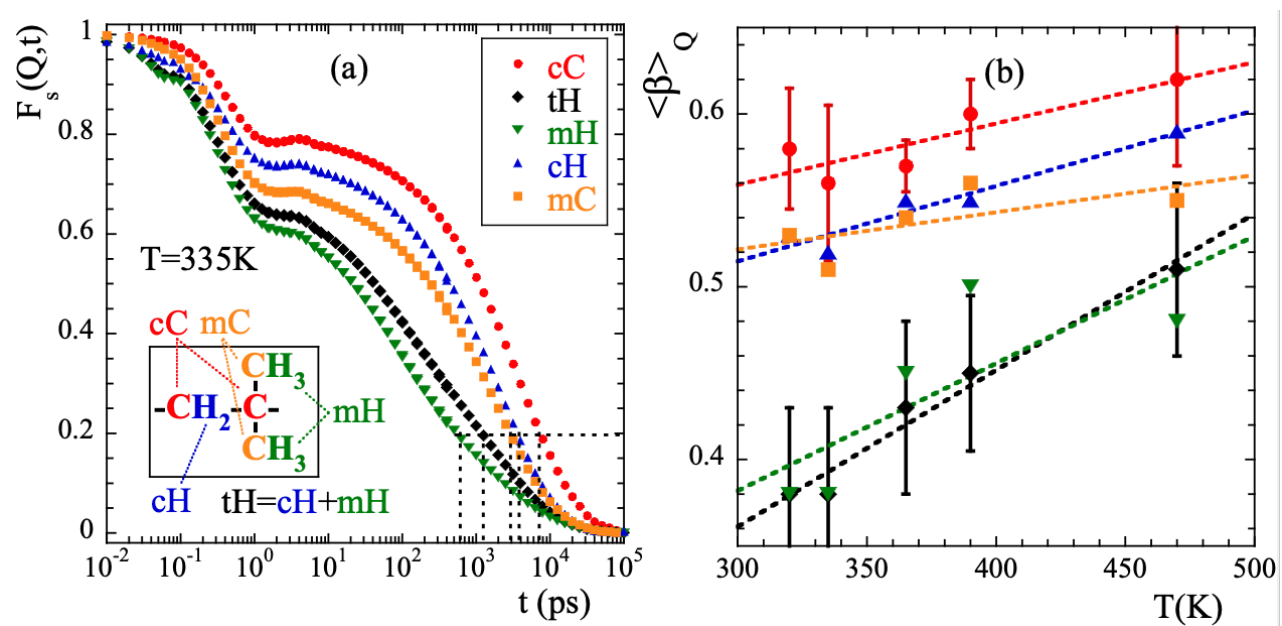

Figure 3. (a) Intermediate scattering function calculated for the different kinds of atoms in PIB at $Q=1.5 \AA^{-1}$ and $335 \mathrm{~K}$. Horizontal dotted line shows reference level for the definition of $\tau_{0.2}$. Inset: Scheme of the PIB monomer illustrating the nomenclature for the different kinds of atoms considered. (b) Temperature dependence of the $Q$-averaged values of the stretching parameter for the different species. Symbols as in (a). For the cases of $\mathrm{cC}$ and $\mathrm{tH}$, the vertical bars show the range over which the $Q$-dependent values span at each temperature. Lines are linear regression fits to guide the eye.

We have characterized the second decay (times longer than 2 ps) of the incoherent scattering functions of the different atomic species by using the commonly invoked stretched exponential or Kohlrausch-Williams-Watts functional form [1]:

$$
F_{S}(Q, t)=A \exp \left[-\left(\frac{t}{\tau_{w}(Q, T)}\right)^{\beta}\right] .
$$

Here $A$ is the amplitude accounting for the decay of the correlations in the microscopic regime, $0<\beta \leq 1$ is the stretching exponent characterizing the deviations with respect to a single exponential decay and $\tau_{w}(Q, T)$ is the characteristic time. In a first step, we fitted Equation (13) to the simulated curves allowing the three parameters to float. For a given species and temperature, we did not find a systematic variation with $Q$ of the values obtained for the $\beta$-parameter. Therefore, we calculated the $Q$-averaged values of this parameter $\langle\beta\rangle_{Q}$ and considered them as representative for each temperature and kind of atom. They are presented in Figure $3 \mathrm{~b}$. The highest values of this parameter are found for $\mathrm{cC}$ and the lowest for $\mathrm{mH}$ (and $\mathrm{tH}$ ). A tendency to increase with increasing 
temperature is observed for all the species, in particular for $\mathrm{mH}$. In a second step, we fitted again Equation (13) by fixing the values of the $\beta$-parameters to the average values $\langle\beta\rangle_{Q}$. The descriptions obtained were reasonably good. Given the change of the shape parameter with temperature and considered species, in the following we will discuss the results corresponding to the characteristic times in terms of the average characteristic times $\langle\tau\rangle$, that in the case of KWW functions are related with $\tau_{w}$ through the expression $\langle\tau\rangle=\Gamma(1 / \beta) \tau_{w} / \beta$. For $365 \mathrm{~K}$, Figure 4 shows the $Q$-dependence of $\langle\tau\rangle$ obtained for the different species. In the low- $Q$ limit (large length scales), all the times roughly coincide. With increasing $Q$ they decrease, showing a certain curvature in the log-log representation. The values corresponding to $\mathrm{CC}, \mathrm{mC}$ and $\mathrm{cH}$ tend to coincide in the high- $Q$ range (local length scales) where those of $\mathrm{mH}$ remain faster.

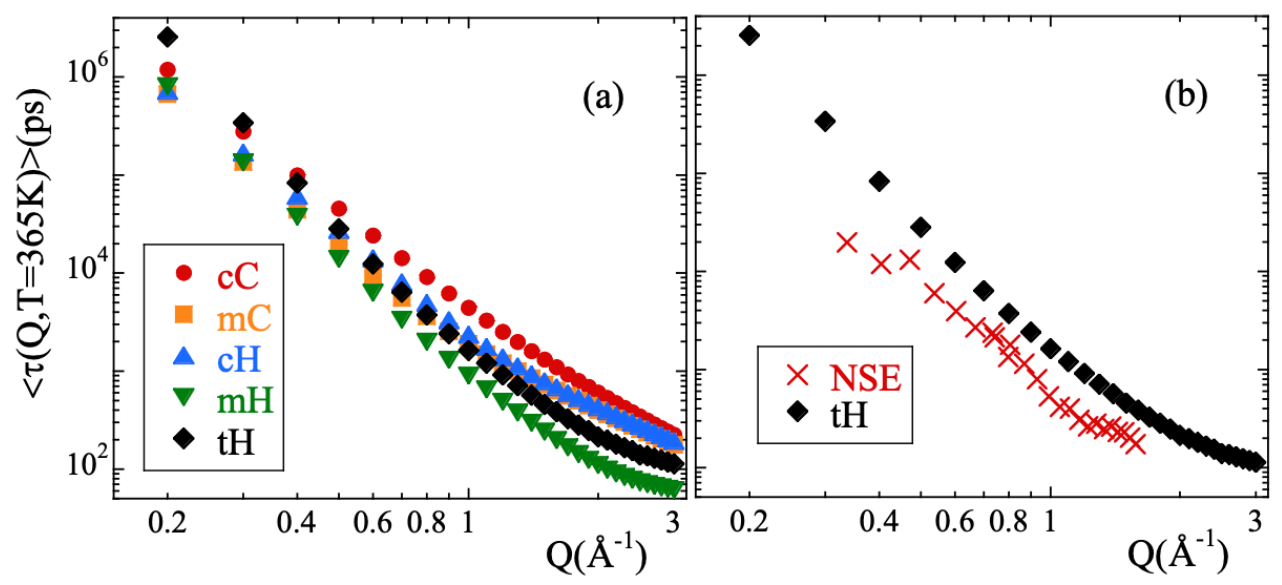

Figure 4. Momentum transfer dependence of the average characteristic times obtained at $365 \mathrm{~K}$ for (a) the different atomic species and (b) for the total hydrogens compared with the experimental NSE results reported in Ref. [13].

Figure 5a-e show the $Q$-dependence of the average characteristic times for the different temperatures investigated. Each panel corresponds to a given atomic species. In the low- $Q$ region, in all cases the data follow well a power law increase as

$$
\tau \propto Q^{-2 / \beta}
$$

(see dotted lines). Here, the $\beta$-value used at each temperature is the $Q$-averaged value shown in Figure 3b. This observation implies the Gaussian form of the scattering function (Equation (5) in such a Q-range [4], as can be deduced when Equation (14) is considered together with Equation (13). Note that since the value of the stretching parameter used in the fits is common for all $Q$-values, considering $\tau_{w}$ or $\langle\tau\rangle$ is equivalent for the discussion of the $Q$-dependence of the characteristic times. The only difference is a prefactor.

The asymptotic Gaussian laws $\tau \propto Q^{-2 / \beta}$ cease to describe the $Q$-dependence of the characteristic times in a $Q$-range that varies from one species to the other but for a given kind of atom hardly depends on temperature (see shadowed areas in Figure 5). At higher $Q$-values, the $Q$-dependence is weaker than that given by Equation (14), indicating deviations from the Gaussian approximation. Thus, the crossover from Gaussian to nonGaussian behavior is found not only for the total hydrogens (as it was reported from the NSE experiments [13]), but for all kinds of atoms in PIB. 


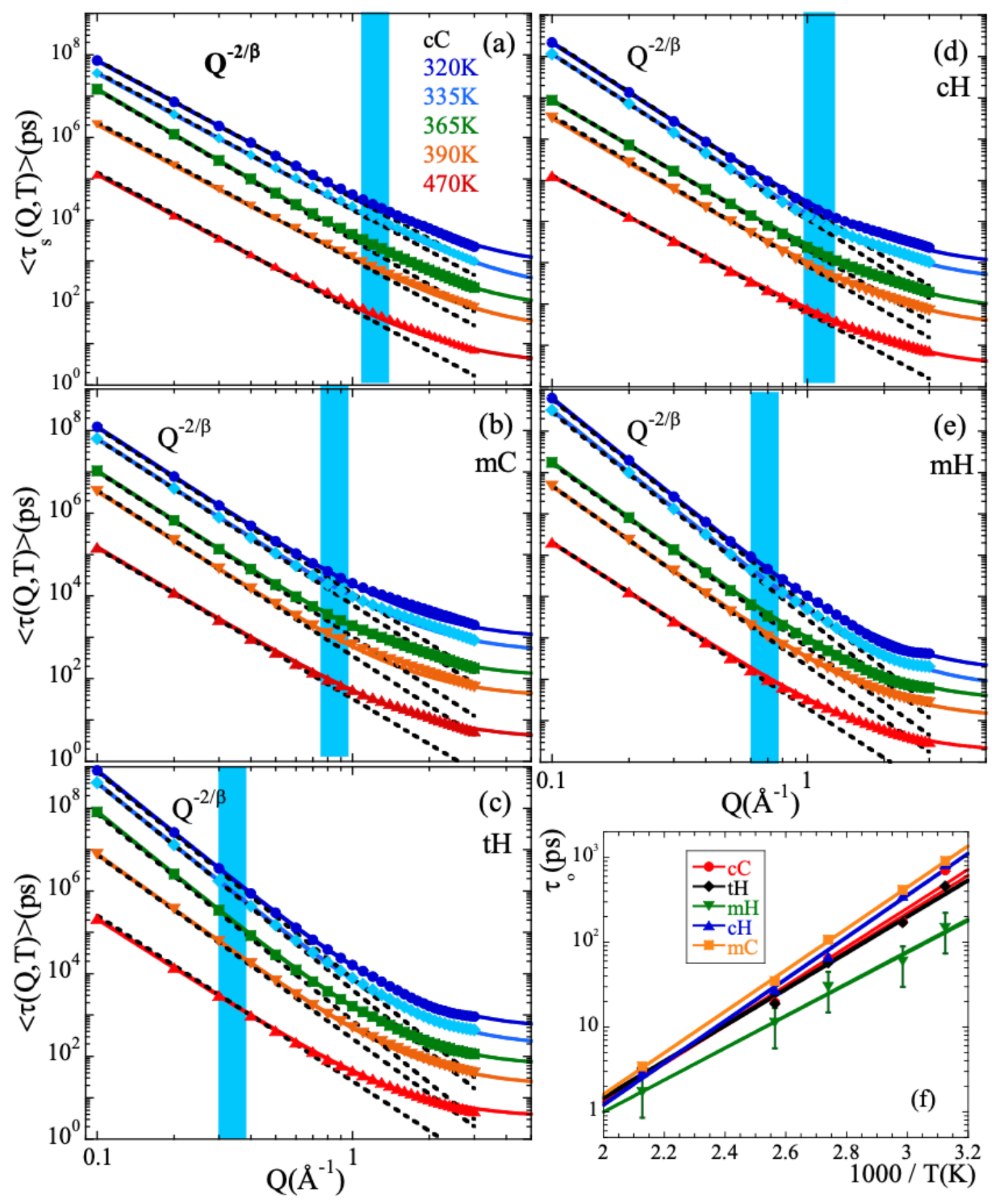

Figure 5. (a-e) Momentum transfer dependence of the average characteristic times obtained for the different temperatures (see color code in Panel (a)) for the different atomic species: main-chain carbons (a); methyl-group carbons (b); total hydrogens (c); main-chain hydrogens (d); methyl-group hydrogens (e). Continuous lines are fits of the anomalous jump diffusion model [Equation (15)]. Dotted lines show the asymptotic Gaussian law [Equation (14)]. In both cases, the values of $\langle\beta\rangle_{Q}$ shown in Figure $3 b$ have been used. Shadowed areas indicate the $Q$-range where deviations from the Gaussian approximation start to develop. (f) Temperature dependence of the elementary characteristic time involved in the anomalous jump diffusion model for the different kinds of atoms in PIB. Representative error bars are shown for the mH species. Solid lines are fits of Arrhenius equations with activation energy $E_{a}^{A J D}$ (see Table 1).

The anomalous jump diffusion model $[14,15]$ has been applied to the different atomic species distinguished in PIB. In this model, an atom remains in a given site for a time $\tau_{0}$, where it vibrates around a center of equilibrium. After $\tau_{0}$, it moves rapidly to a new position. These jumps are assumed to occur with random orientations and their moduli $\ell$ 
are distributed according to a function $f_{o}(\ell)=\frac{\ell}{\ell_{0}^{2}} \exp \left(-\frac{\ell}{\ell_{0}}\right)$ which involves a preferred jump distance $\ell_{0}$. The characteristic time then follows the law

$$
\tau_{w}=\tau_{o}\left(1+\frac{1}{\ell_{o}^{2} Q^{2}}\right)^{\frac{1}{\beta}}
$$

Figure 5 shows as continuous lines the very satisfactory descriptions obtained. Again, we have imposed here the $Q$-averaged values of $\beta$ shown in Figure $3 \mathrm{~b}$. For the different types of atoms, the temperature variation of $\ell_{0}$ and $\tau_{0}$ are represented in Figure S1 of the Supplemental Material (SM) and Figure $5 \mathrm{f}$ respectively. The values of $\ell_{0}$ do not appreciably change with temperature. The $\ell_{0}$-values averaged over all the temperatures investigated are listed in Table 1. For the main-chain atoms, they increase from $\mathrm{cC}$ to $\mathrm{cH}$ (0.3 to $0.4 \AA$, respectively); hydrogens undergo larger jumps than carbons in the decaging process. The value obtained for $\mathrm{cH}$ is comparable to that reported for the main-chain hydrogens in e.g., polyisoprene $(0.42 \AA)$ [14,15]. For methyl-group atoms this length is larger than for the main-chain counterparts and rather similar for both $\mathrm{mH}$ and $\mathrm{mC}(0.50$ and $0.54 \AA$ ). For the total hydrogens, the value obtained for $\ell_{0}$ is largest. This result can be understood considering that in this case the $Q$-dependence of the characteristic times reflects deviations from Gaussian behavior which are a consequence not only of the discrete nature of the underlying mechanism for sublinear diffusion (basic ingredient of the anomalous jump diffusion model) but also of the heterogeneous dynamics developed by $\mathrm{cH}$ and $\mathrm{mH}$. The more pronounced deviations from Gaussian behavior are captured in this simple model by an effectively larger value of the jump distance. A similar situation was discussed for PVE [19], PVME [20], styrene-butadiene-rubber (SBR) and polystyrene (PS) [70]. Additional ingredients were also included to describe the behavior found in complex systems like e.g., confined liquid crystals [71] or proton diffusion in polymer eletrolyte fuel cell membranes [72]. Furthermore, this intrinsic heterogeneity -together with the occurrence of $\mathrm{mH}$-rotations as we will show in the following section-is also the cause of large stretching for $\mathrm{tH}$. On the other hand, the values of $\tau_{0}$ corresponding to $\mathrm{cC}, \mathrm{cH}$ and $\mathrm{mC}$ are rather similar and show nearly identical values of the activation energy $E_{a}^{A J D}$ (see Table 1). For $\mathrm{mH}$ the obtained values are smaller and the activation energy weaker. Thus, methyl-group rotations lead to a quicker delocalization of $\mathrm{mH}$ atoms.

Table 1. Values of the preferred jump length $\ell_{0}$ and activation energy of the characteristic time $\tau_{0}, E_{O}^{A J D}$, involved in the description by anomalous jump diffusion model, for the different atomic species distinguished in PIB.

\begin{tabular}{ccc}
\hline Atomic Species & $\boldsymbol{\ell}_{\boldsymbol{o}}(\AA)$ & $\boldsymbol{E}_{\boldsymbol{o}}^{A J D}(\mathbf{e V})$ \\
\hline $\mathrm{cC}$ & $0.32 \pm 0.06$ & $0.44 \pm 0.13$ \\
$\mathrm{mC}$ & $0.54 \pm 0.03$ & $0.47 \pm 0.11$ \\
$\mathrm{cH}$ & $0.41 \pm 0.03$ & $0.47 \pm 0.13$ \\
$\mathrm{mH}$ & $0.50 \pm 0.03$ & $0.32 \pm 0.10$ \\
$\mathrm{tH}$ & $0.57 \pm 0.03$ & $0.42 \pm 0.13$ \\
\hline
\end{tabular}

It is noteworthy that in essence the anomalous jump diffusion model incorporates the ingredient of caging by considering a distribution of jumps underlying the diffusive-like motion of atoms in the $\alpha$ process. The cage effect is the main concept involved also in the mode coupling theory (MCT) [73,74]. The applicability of MCT to the case of PIB was explored in a previous work [75]. The localization length $r_{s c}$ involved in the MCT was found to be $r_{s c}=0.30 \AA$ for $\mathrm{CC}$ and $r_{s c}=0.50 \AA$ for $\mathrm{mH}$. These values are very similar to those obtained in the anomalous jump diffusion model for the preferred jump distance. We note that this was also the case for $\mathrm{cH}$ in PI [15].

From the simulations we thus confirm the experimental observation of stretching of $F_{s}(Q, t)$, its Gaussian behavior at low $Q$ s and deviations at higher $Q$-values reported from 
experiments. The larger window covered, the better statistics of the data and the capability of isolating the incoherent contribution has made it possible to determine with more accuracy the value of the shape parameter and the limits of the Gaussian approximation. It is meaningful to compare the outcome of the analysis of the NSE results [13] and those of the simulations. In the interpretation of the NSE data, it is assumed that $S_{N S E}(Q, t)=F_{S}^{t H}(Q, t)$. Figure $1 \mathrm{~b}$ shows that this is a very good approximation, though the coherent contribution can be noticeable in the $Q$-range of the first structure factor peak $\left(\approx 1 \AA^{-1}\right)$. Figure $4 \mathrm{~b}$ compares the average relaxation times for $\mathrm{tH}$ from the simulations with those reported from the experiments. The latter are systematically faster. This is mainly due to the choice of the shape parameter $\beta$ : the NSE results were fitted by assuming a fixed value of 0.55 deduced from the analysis of collective data. This value is sensitively larger than that obtained from the simulations (see Figure $3 b$ ). Such relatively small values of $\beta$ are due to the heterogeneous motions of $\mathrm{cH}$ and $\mathrm{mH}$, i.e., ultimately to the occurrence of methyl-group rotations. These motions are discussed in more detail in the following.

\subsection{Methyl Group Dynamics}

In the high temperature range here investigated, the $\mathrm{mH}$ are expected to undergo classical hopping in a rotational potential. We have found indications for the rotations in the low values of the $\beta$-parameter used to describe the second decay of $F_{S}(Q, t)$ for $\mathrm{mH}$ and in the faster corresponding times observed in the high $Q$-range with respect to those of the other species. However, since the segmental dynamics ( $\alpha$-relaxation) is superimposed, a two-steps decay of $F_{S}(Q, t)$ after the microscopic regime is not observed for $\mathrm{mH}$ in the whole temperature range investigated (see e.g., Figure 3a), preventing a direct characterization of these rotational motions.

Exploring the correlation functions directly in real space (the self-part of the van Hove correlation functions $G_{\mathcal{S}}(r, t)$ ) can be of utmost help to characterize the atomic motions. For the two extreme temperatures here investigated, Figure 6 represents these functions for $\mathrm{mH}$ at different times. At $470 \mathrm{~K}$, the features of the probability distribution function correspond to a diffusive-like process: increasing broadening and shift toward larger distances with increasing time. At $320 \mathrm{~K}$ some hints of a jump-like process with the appropriate characteristic length $\left(r_{H H}=1.78 \AA\right.$ is the distance between the hydrogens in the methyl group) could be envisaged in the range $100 \ldots 1000$ ps. However, the overall behavior is also rather close to a diffusional process.

Thus, in order to characterize the methyl-group rotations the data have to be 'cleaned out' from the diffusive-like motions of the $\alpha$-relaxation. In a first approximation, it could be assumed that both kinds of motions occur simultaneously and are statistically independent. We have checked this hypothesis for PIB. Under such conditions, the incoherent scattering functions for methyl group hydrogens $F_{S}^{m H}(Q, t)$ would be given by the product of the incoherent scattering functions corresponding to the purely rotational motion and to the $\alpha$-relaxation process. The latter can be approximated with the incoherent scattering function calculated for main-chain hydrogens $F_{s}^{c H}(Q, t)$. This approach was successfully used in the case of PI [76] and is in fact inspired in the way an experimentalist would face this kind of problem, provided the availability of NS measurements on fully protonated and partially deuterated samples. This allows to obtain in a straightforward way the deconvoluted rotational function by simple division of the two known functions, $F_{s}^{\text {Deconv }}(Q, t)=F_{s}^{m H}(Q, t) / F_{s}^{c H}(Q, t)$. If the invoked assumptions are correct, consistent results should be obtained. First of all, its functional form should be that of a localized motion. This implies that its long-time limit should be a constant value, the so-called Elastic Incoherent Incoherent Structure Factor (EISF). The EISF gives account for the geometry of the motion. Figure 7 shows the results of applying the deconvolution approach to PIB. A pronounced first decay in the microscopic regime below 1 ps is seen, that is due to the larger motional amplitude at shorter times of the $\mathrm{mH}$ with respect to the $\mathrm{cH}$. A second decay towards a plateau is clearly visible at high $Q$-values for the low temperature (Figure 7a), that is reached at about 2 ns. At longer times, mainly in the low- $Q$ range, the obtained 
curves do not really show a constant value, as it should happen if motions are independent. At the highest temperature of $470 \mathrm{~K}$ (Figure $7 \mathrm{~b}$ ), no plateau can even be identified in the curves. We can thus infer that the initial assumption of statistical independence fails. If the segmental contribution is approximated by considering the scattering function of main-chain carbons instead of that of main-chain hydrogens, the deconvolution does not work better. The conclusion is that the deconvolution assumption implying the statistical independence of $\alpha$-process and methyl-group rotations fails for PIB, not only for long times and high temperatures, but also at the lowest temperatures investigated at intermediate times and intermolecular length scales, as we show in detail in the SM.
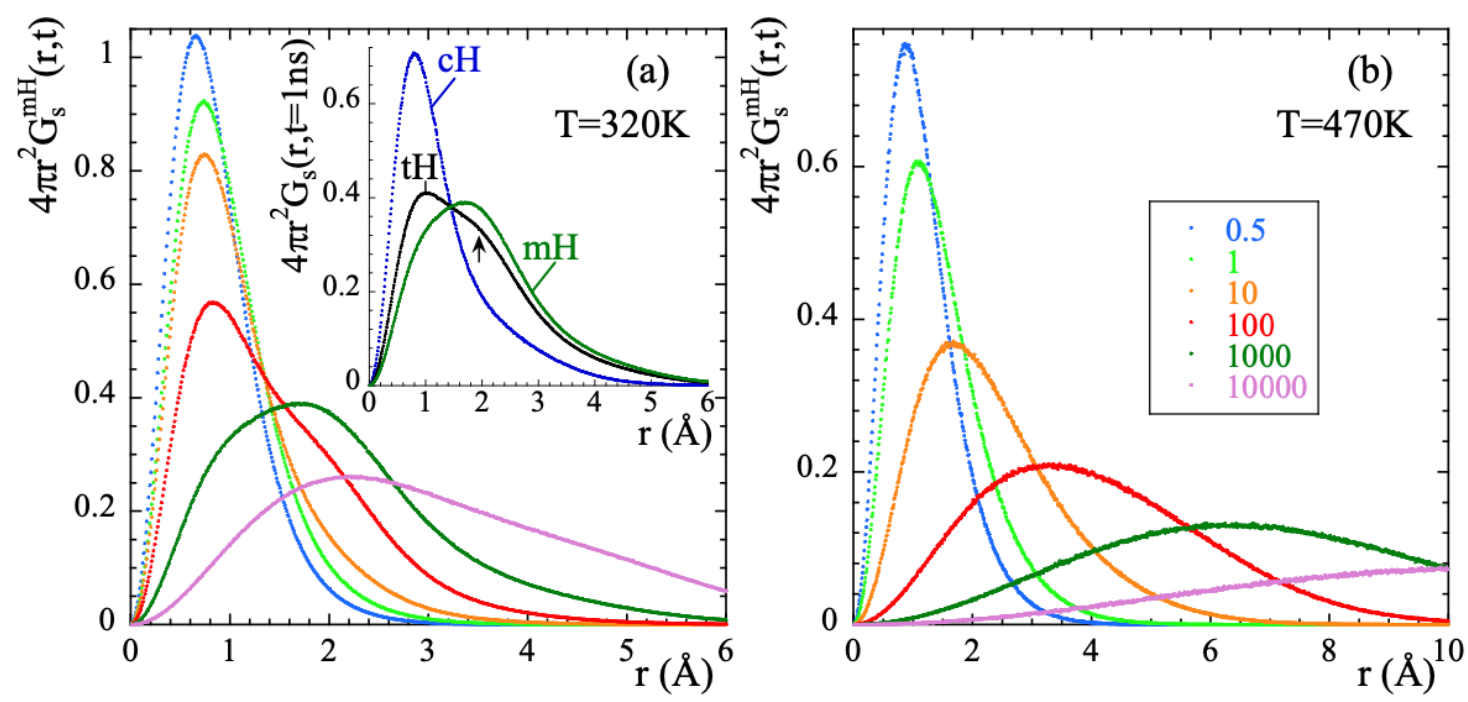

Figure 6. Radial probability distribution function calculated for methyl-group hydrogens at $320 \mathrm{~K}$ (a) and $470 \mathrm{~K}$ (b) at the different times (in ps) indicated in (b). For $t=1000 \mathrm{ps}$, the insert in (a) compares this function for methyl-group hydrogens, main-chain hydrogens and total hydrogens. The arrow marks the location of a second peak (hump) in the function corresponding to tHs (see the text).
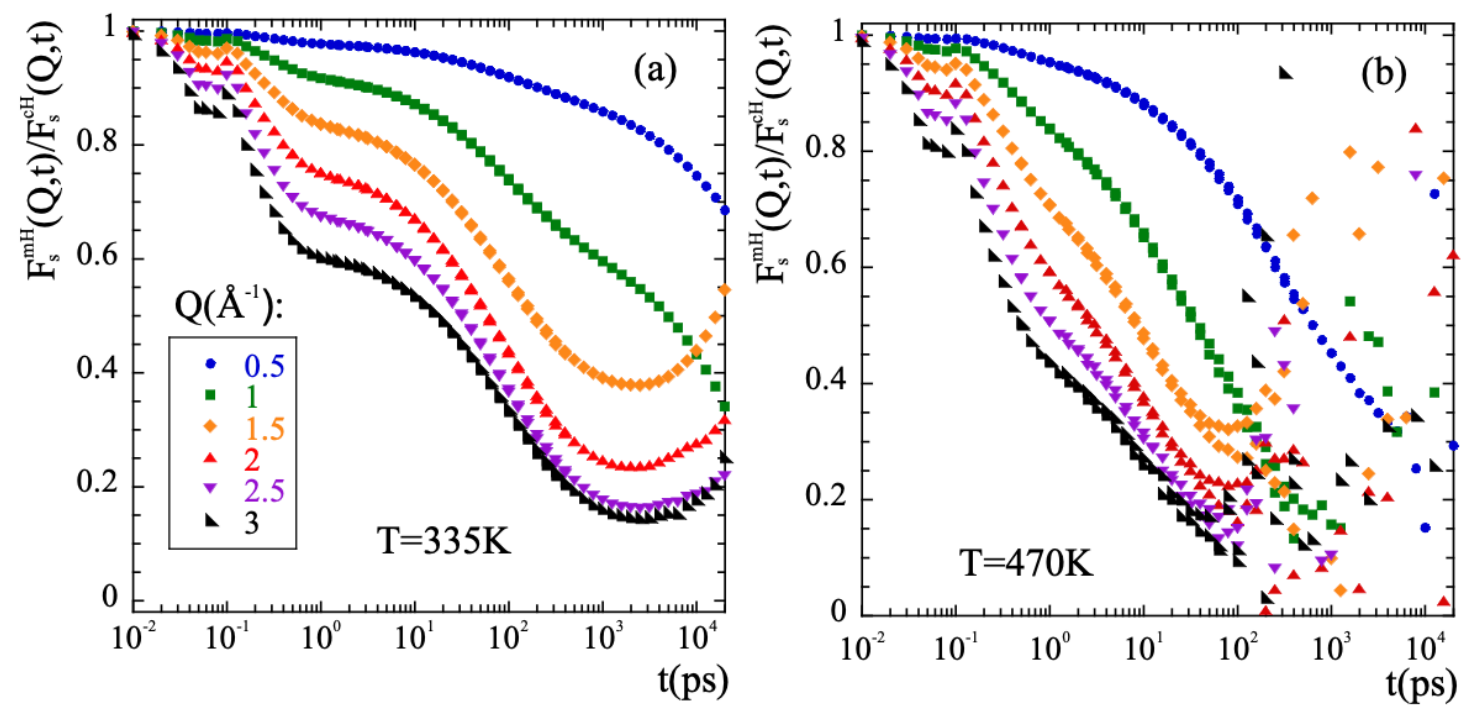

Figure 7. Result of dividing the intermediate scattering functions corresponding to methyl-group hydrogens and main-chain hydrogens at the $Q$-values indicated in (a) for $T=335 \mathrm{~K}(\mathbf{a})$ and $T=470 \mathrm{~K}(\mathbf{b})$. 
In order to characterize the rotational dynamics of the methyl groups in PIB, we have calculated from the simulations the correlation functions corresponding to the relative motions of $\mathrm{mH}$ with respect to the $\mathrm{mC}$ to which they are linked. Figure $8 \mathrm{a}$ shows as an example the intermediate scattering function $F_{s}^{\text {rel }}(Q, t)$ calculated at $335 \mathrm{~K}$ and different $Q$-values. We applied the rotational rate distribution model (RRDM) $[41,77,78]$ (see SM) to describe this function. The RRDM assumes the presence of a Gaussian distribution of potential barriers for methyl group rotations as consequence of the disorder. The only free parameters are the width $\sigma$ and the center $\tau_{o}^{M G}$ of the log-normal distribution function of characteristic times for rotation $H(\log \tau)$. In addition, obviously, an amplitude $A$ accounting for the microscopic contribution has to be used. The RRDM with $Q$-independent $\tau_{o}^{M G}$ and $\sigma$ parameters provides the descriptions shown in Figure 8a as dotted lines. They are very good for $Q$-values in the range $Q \leq 1.5 \AA^{-1}$ and show some deviations at higher $Q$-values; in particular, the theoretical EISF:

$$
E I S F=\frac{1}{3}\left(1+2 \frac{\sin \left(Q r_{H H}\right)}{Q r_{H H}}\right) .
$$

seems to underestimate the values obtained from the simulations. Allowing free $A$, EISF, $\tau_{o}^{M G}$ and $\sigma$ parameters the fits reproduced very well all the results (solid lines in the figure). Figure $8 \mathrm{~b}$ shows the values obtained for the EISF parameter at $335 \mathrm{~K}$ (empty diamonds) as compared with the theoretical prediction Equation (16). Figure 9 displays $\tau_{0}^{M G}$ and $\sigma$ for all temperatures investigated.
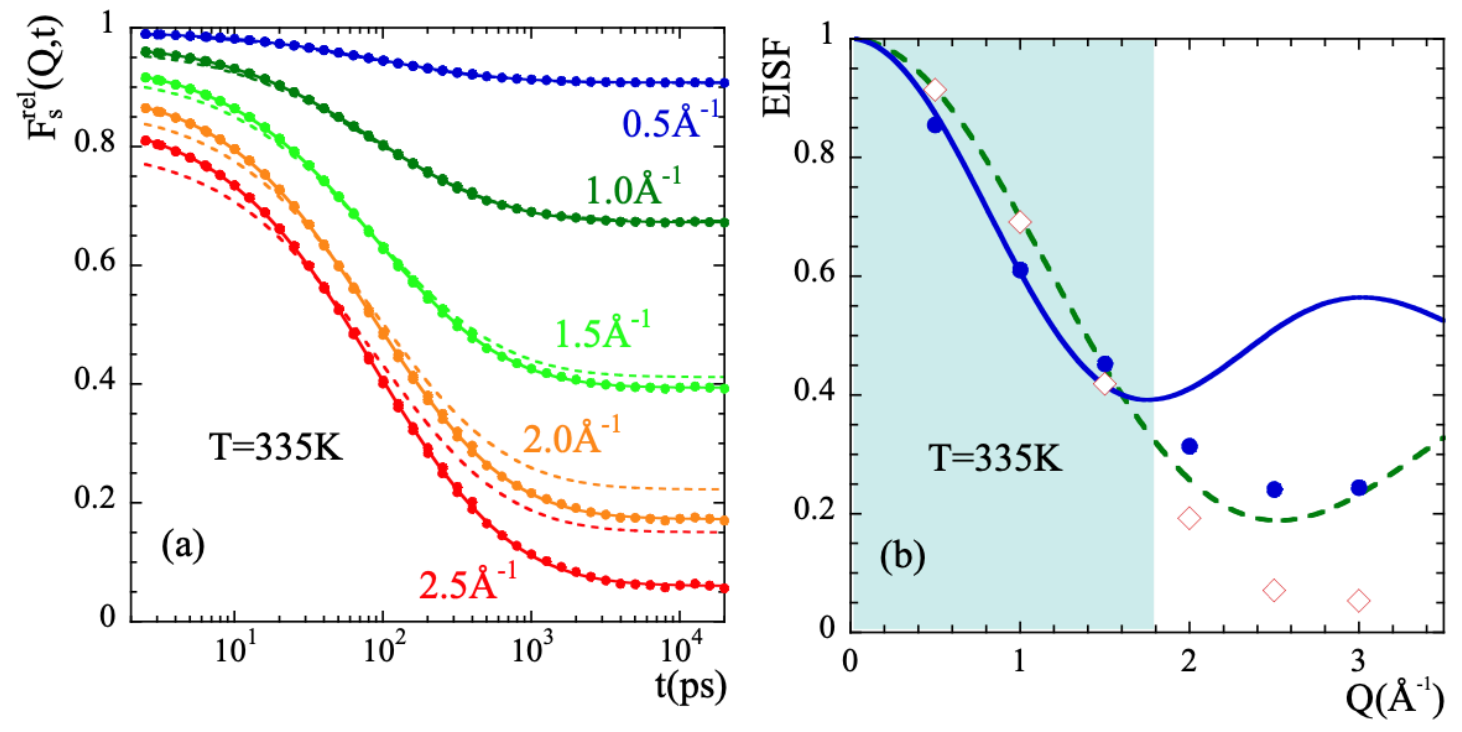

Figure 8. (a) Intermediate scattering function of methyl-group hydrogens corresponding to their motion relative to the methyl-group carbon $F_{S}^{r e l}(Q, t)$ at the $Q$-values indicated. The dotted lines are fits with the RRDM imposing the theoretical 3-fold EISF (dotted line in (b)) and $Q$-independent $\sigma$ and $\tau_{o}^{M G}$, while in the solid lines free A, EISF, $\sigma$ and $\tau_{o}^{M G}$ have been allowed. The such resulting EISF values are shown in (b) (diamonds). There, the 'apparent EISF' obtained from the deconvolution approach (see the text) is shown by the solid circles. The continuous line is a description of these data within the experimental window explored in Ref. [34] (shadowed area) in terms of Equation (18). The resulting $d$-value is $2.6 \AA$. In all cases the temperature is $335 \mathrm{~K}$. 

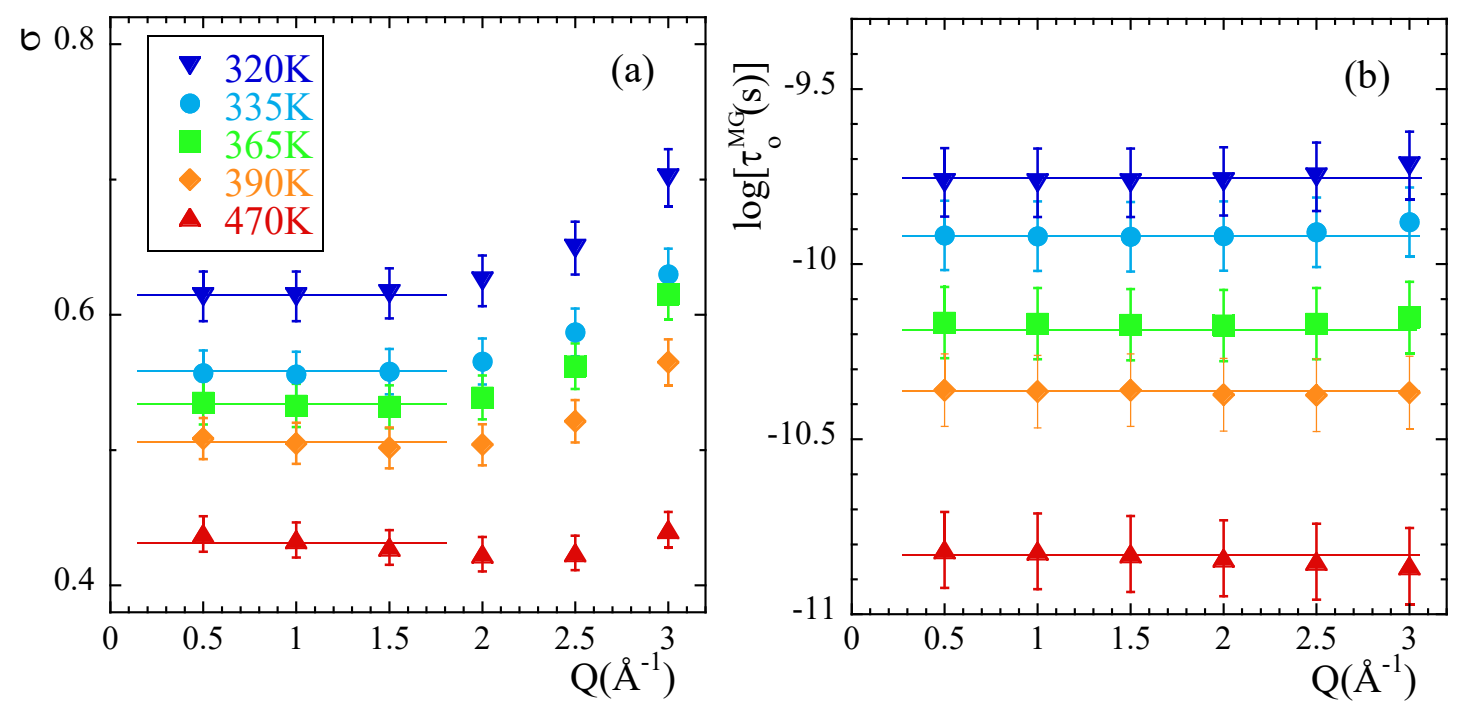

Figure 9. Momentum transfer dependence of the RRDM parameters $\sigma(\mathbf{a})$ and $\tau_{o}^{M G}(\mathbf{b})$ obtained from the fit of $F_{s}^{\text {rel }}(Q, t)$ at the temperatures indicated in (a). Lines show the values considered as representative for these parameters.

As can be seen in Figure 8b, the EISF-values show a more pronounced decay than that corresponding to a 3-fold potential for $Q$-values $>1.5 \AA^{-1}$ approx. Obviously, the geometry of the motions carried out by $\mathrm{mH}$ is not fully describable by a rigid rotor in a three-fold potential. The $\mathrm{mH}$ motions lead to almost a complete decay of EISF at high $Q$-values.

Now let us focus on the parameters describing the distribution function of rotational times. As expected for a localized motion, the characteristic time $\tau_{0}^{M G}$ is $Q$-independent (see Figure $9 \mathrm{~b}$ ). The width of the distribution function $\sigma$ also displays an approximately constant value for $Q<2 \AA^{-1}$ but a slight tendency to increase is observed at higher $Q$-values. We have thus considered the average value of $\sigma$ in the low- $Q$ region as that representing the actual width of the distribution of rotational times at a given temperature (lines in Figure 9a). The results corresponding to $\sigma$ are inversely proportional to temperature, implying that the data are compatible with an underlying Gaussian distribution of activation energies $E_{a}^{M G}$ (see $\mathrm{SM}$ ). The value obtained for $\sigma_{E}$ is $38.5 \mathrm{meV}(447 \mathrm{~K})$. The average activation energy $\left\langle E_{a}^{M G}\right\rangle$ is obtained from the Arrhenius fit of $\tau_{0}^{M G}$,

$$
\tau_{o}^{M G}=\tau_{\infty} \exp \left(\frac{\left\langle E_{a}^{M G}\right\rangle}{K_{B} T}\right)
$$

leading to $\left\langle E_{a}^{M G}\right\rangle=0.21 \mathrm{eV}(2452 \mathrm{~K})$ and $\tau_{\infty}=0.08 \mathrm{ps}$. Compared with other glass-forming systems [41], PIB shows a very large value of $\left\langle E_{a}^{M G}\right\rangle$ (typical values are around $1000 \mathrm{~K}$ ) but comparable to that displayed by methyl groups located in bisphenol-A moeties (like in polycarbonate, polysulfone and phenoxy [41]).

The direct insight of the atomic motions in real space facilitated from the simulations can help to unveil more details of $\mathrm{mH}$ dynamics. Figure 10 shows the radial probability distribution function calculated from the $\mathrm{mH}$ trajectories relative to those of $\mathrm{mC}$. At times below $\approx 1$ ps the probability shows only one clear maximum broadened by vibrational motions. With increasing time a second maximum is clearly developed which position is separated from that of the first one by the distance $r_{H H}=1.78 \AA$. The relative populations of the two peaks continuously change with increasing time. At the longest times represented, the radial probability distribution function continuously increases with distance in the physically accessible spatial range. This behavior is qualitatively different from that expected for the 3 -fold model of a rigid rotor. 
This observation, together with the previously commented increase of $\sigma$ and decrease of EISF detected at high $Q$-values could be attributed to a superimposed translational component of $\mathrm{mH}$ atoms. In order to proof this hypothesis, we generated some random distributions of the accessible positions of such a proton (accordingly to the geometric configuration of a methyl group) allowing for some minor fluctuations for the methyl carbon-proton bond length and also for some stochastic vibrations around the three minima of the methyl rotor. The result is depicted by the filled circles in the insert of Figure 10 that is the one to be expected for a (semi-)rigid rotor of the corresponding geometry with a symmetry axis which direction is fixed in space. Now, if one releases this constraint and allows the axis of the rotor to vary its direction in space, the curve with empty symbols is obtained, which is very similar to those obtained in Figure $10 \mathrm{~b}$ for the longest time.

To determine the time at which the probability distribution function $4 \pi r^{2} G_{s}^{r e l}(r, t)$ deviates from the expected behavior for a rotor with fixed axis, we have considered the value of this function at $r=r_{H H} / 2=0.89 \AA$. This parameter represents the way how the minimum in the probability distribution function is getting populated. In principle, its value should remain constant in time once the stationary regime for rotations has been achieved. Figure $11 \mathrm{~b}$ shows the results obtained at $335 \mathrm{~K}$. The time at which deviations start is about 200 ps. For comparison, Figure 11a shows with circles the mean squared displacement $\left\langle r^{2}(t)\right\rangle$ and the non-Gaussian parameter $\alpha_{2}(t)$ calculated for main-chain carbons at the same temperature. A characteristic time for the processes developed in the cageing regime $t^{\star}$ is usually defined as that where the $\alpha_{2}$-parameter reaches its maximum. For main-chain carbons, $t^{\star}$ is very close to 200 ps at this temperature. Thus, the nonGaussian events undergone by backbone atoms seem to be at the origin of the peculiarities observed for methyl-group rotations.

The inverted triangles in Figure 11a show the results corresponding to the relative motion of $\mathrm{mH}$ with respect to $\mathrm{mC}$ at $335 \mathrm{~K}$. At this temperature, for $t \geq 1 \mathrm{~ns} \mathrm{mH}$ explore the full available space around $\mathrm{mC}$. There, the function $F_{s}^{\text {rel }}(Q, t)$ reaches the limit $t \rightarrow \infty$ (see Figure 8 b). From the comparison between the mean squared displacements in Figure 11a we can see that the development of the localized motions by the methyl groups' hydrogens takes place just during the occurrence of the decaging events in the backbone atoms. At longer times, in the region where $\left\langle r_{r e l}^{2}(t)\right\rangle$ is constant, $\left\langle r_{c C}^{2}(t)\right\rangle$ follows the sublinear increase $\left\langle r^{2}(t)\right\rangle \propto t^{\beta}$ characteristic for the $\alpha$-relaxation.

We can now consider the information provided by the distribution functions of rotational times for $\mathrm{mH}, H(\log \tau)$. From these functions, the values of the average residence time for methyl-group hydrogens $\tau_{R}^{O}$ can be obtained for the different temperatures (since $\tau_{R}$ is related with $\tau$ through $\tau_{R}=3 \tau / 2$, see $S M, \tau_{R}^{o}=3 \tau_{0} / 2$ ). This time is represented in the insert of Figure $11 \mathrm{~b}$ together with the characteristic time for decaging processes of backbone carbons $t_{c C}^{\star}$. Both times almost perfectly agree for all temperatures. Thus, methyl-group rotations and main-chain decaging are clearly coupled for PIB in the temperature range investigated. A similar conclusion was deduced from the MD-simulations reported in Ref. [38]. Finally, in the same figure we have also included (dashed line) the characteristic times reported for the so-called $\delta$-relaxation from NMR [36,38] and ESR [37] investigations. The $\delta$-process had been assigned by Slichter [36] to originate undoubtedly from a methyl group rotation. The excellent agreement found between our results and this process definitely supports our analysis.

Furthermore, interestingly, we note that at short times (around $0.08 \mathrm{ps}$ ) the $\left\langle r^{2}(t)\right\rangle$ and $\alpha_{2}(t)$ functions calculated for the relative motions of $\mathrm{mH}$ with respect to $\mathrm{mC}$ display marked peaks suggesting a kind of 'recoil' effect (see Figure 11a). This feature occurs just when the second maximum in the $4 \pi r^{2} G_{s}^{r e l}(r, t)$ function starts to be populated (see Figure 10a), coinciding with the value of the prefactor $\tau_{\infty}$ in Equation (17). 

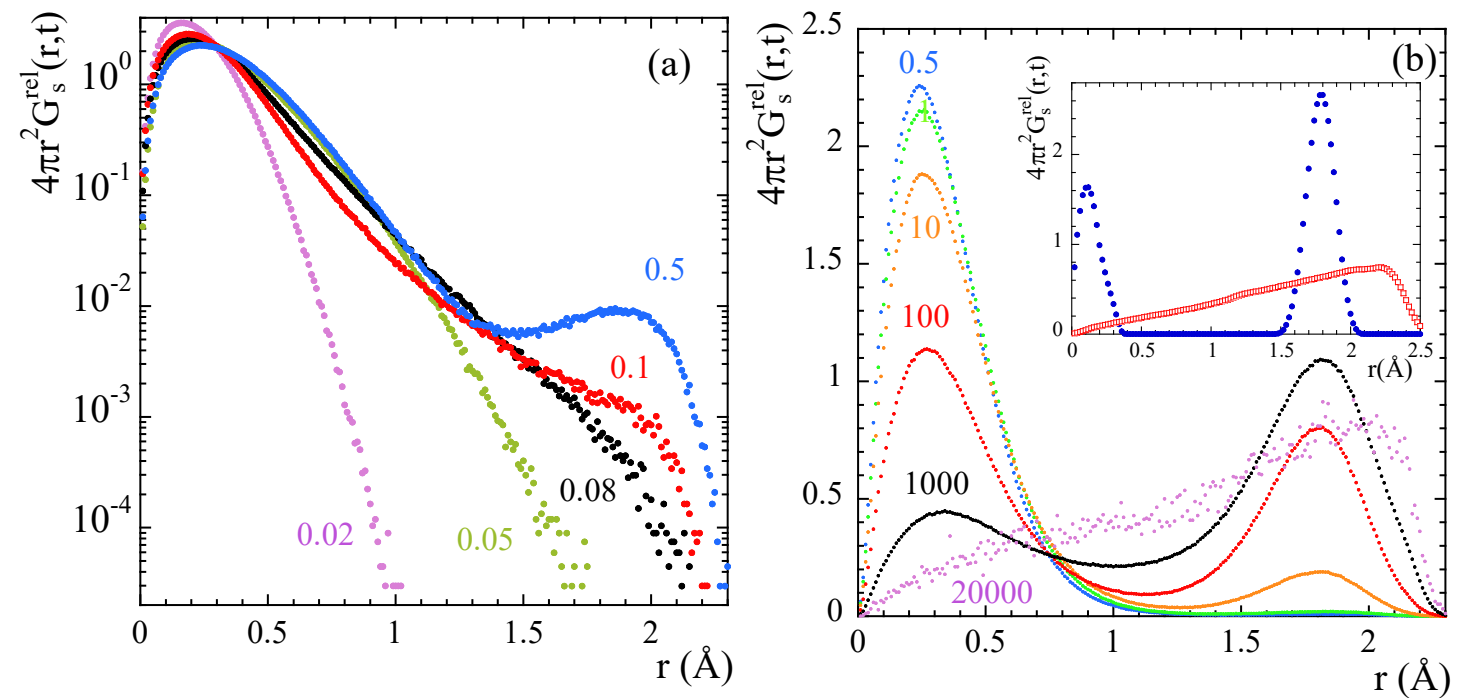

Figure 10. Radial probability distribution function of $\mathrm{mH}$ atoms relative to $\mathrm{mC}$ at $335 \mathrm{~K}$ and different times. Results in (a) are plotted in a logarithmic scale to enhance the subtle differences occurring at the short times shown. Inset in (b): Radial probability distribution function of $\mathrm{mH}$ atoms relative to $\mathrm{mC}$ calculated for a (semi-)rigid rotor (blue dots) and the same rotor with varying direction of the axis (red squares) (see text).

We now address another manifestation of methyl-group dynamics in NS experiments, namely the presence of librational peaks in the inelastic spectra. A peak in the VDOS reveals the energy of the transition between the ground and first excited level $E_{01}$ (see $\mathrm{SM}$ ). We have calculated the VDOS corresponding to $\mathrm{mH}, \mathrm{cH}$ and $\mathrm{tH}$ [see Figure 12a]. In this way, the 'selective deuteration' invoked by Adams et al. [42] was realized. In the region where the NS experiments showed the broad peak for $\mathrm{tH}$ we can clearly distinguish two peaks. The first (and mean) peak indeed corresponds to a $\mathrm{mH}$ peak (presumably that corresponding to $\left.E_{01}\right)$. The second peak of the tH-VDOS at about $50 \mathrm{meV}$, which in the experiments was not really resolved from the first one, is also mainly due to a pronounced peak in the VDOS of $\mathrm{mH}$, but contains additional $\mathrm{cH}$-contributions in the range $40-55 \mathrm{meV}$. We note that a double-peak structure in the range $\approx 34-44 \mathrm{meV}$ was also observed in the VDOS of polysulfone, polycarbonate and phenoxy [41]. Thus, there are striking similarities in the behavior of PIB and those systems, regarding both, the presence of a double peak in this energy region and the above commented unusually large value of $\left\langle E_{a}^{M G}\right\rangle$ (and accordingly, of $\left.\left\langle E_{01}\right\rangle\right)$.

The VDOS of the carbons in the system has also been calculated, distinguishing even the four different carbons. The peak at about $40 \mathrm{meV}$ in the VDOS of $\mathrm{mH}$ appears as main signature of the VDOS in $\mathrm{mC}$. A peak at about $50 \mathrm{meV}$ is present in the VDOS of all the carbon species, and is specially pronounced in the methylene $\mathrm{cC}$. Thus, the appearance of a second peak in the VDOS of $\mathrm{mH}$ at about $50 \mathrm{meV}$ seems to be a consequence of the strong coupling of $\mathrm{mH}$ and $\mathrm{cC}$ dynamics, as proposed by Adams et al. [42] and is consistent with our conclusions from the analysis of the rotational dynamics of $\mathrm{mH}$ at high temperature.

As pointed out in Ref. [42], the peak attributed to methyl-group librations is rather broad. In principle, such broadening could be understood as consequence of the disorder in the glassy material. In fact, this ingredient has already been taken into account analysis of the $F_{s}^{r e l}(Q, t)$ functions by invoking the RRDM. From the obtained distribution of activation energies $f\left(E_{a}^{M G}\right)$ and using the proper relationships (see $\mathrm{SM}$ ) the corresponding distribution function of librational energies $F\left(E_{01}\right)$ can be obtained under the assumption of an underlying 3-fold potential. The result is included in Figure 12a. Though the deduced average value $\left\langle E_{01}\right\rangle=36 \mathrm{meV}$ is slightly shifted toward lower energies with respect to the simulated one, the broadening is well reproduced. The discrepancy observed for $\left\langle E_{01}\right\rangle$ could be due to the fact that the analysis of $F_{s}^{\text {rel }}(Q, t)$ was made for temperatures above $T_{g}$. 
There, the potential 'seen' by the methyl groups could be slightly different from that in the glassy state, due to the softening of the non-bonded interactions at high temperature.

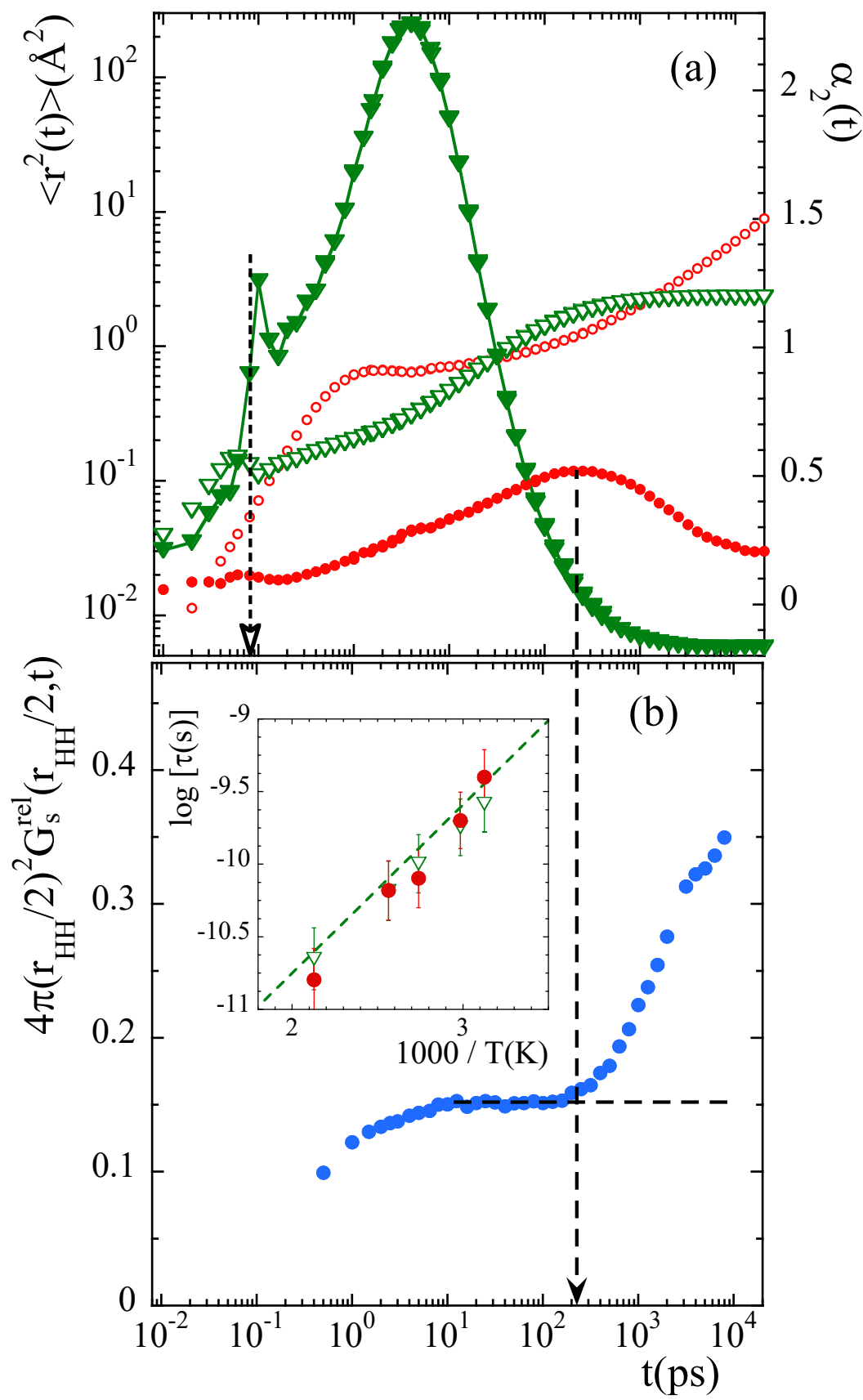

Figure 11. (a) Mean squared displacement (empty symbols) and non-Gaussian parameter (filled symbols) of main-chain carbons (circles) and relative motions of methyl-group hydrogens with respect to the methyl-group carbon (triangles). Vertical dotted arrow marks the value of $\tau_{\infty}$ in Equation (17). (b): Time dependence of the value of the function $4 \pi r^{2} G_{S}^{r e l}(r, t)$ evaluated at $r=r_{H H} / 2$. The horizontal dashed line shows the expected asymptotic behavior in the stationary state of rotations, and the vertical dashed arrow the time at which the data depart from such a behavior. All results correspond to $335 \mathrm{~K}$. Insert: Arrhenius plot compiling the average residence time for methyl-group rotational motions $\tau_{R}^{o}$ (empty triangles), the characteristic time for the maximum of the non-Gaussian parameter $t^{\star}$ corresponding to main-chain carbons (filled circles) and the $\delta$-process reported from NMR [36] and ESR [37] investigations (dashed line). 

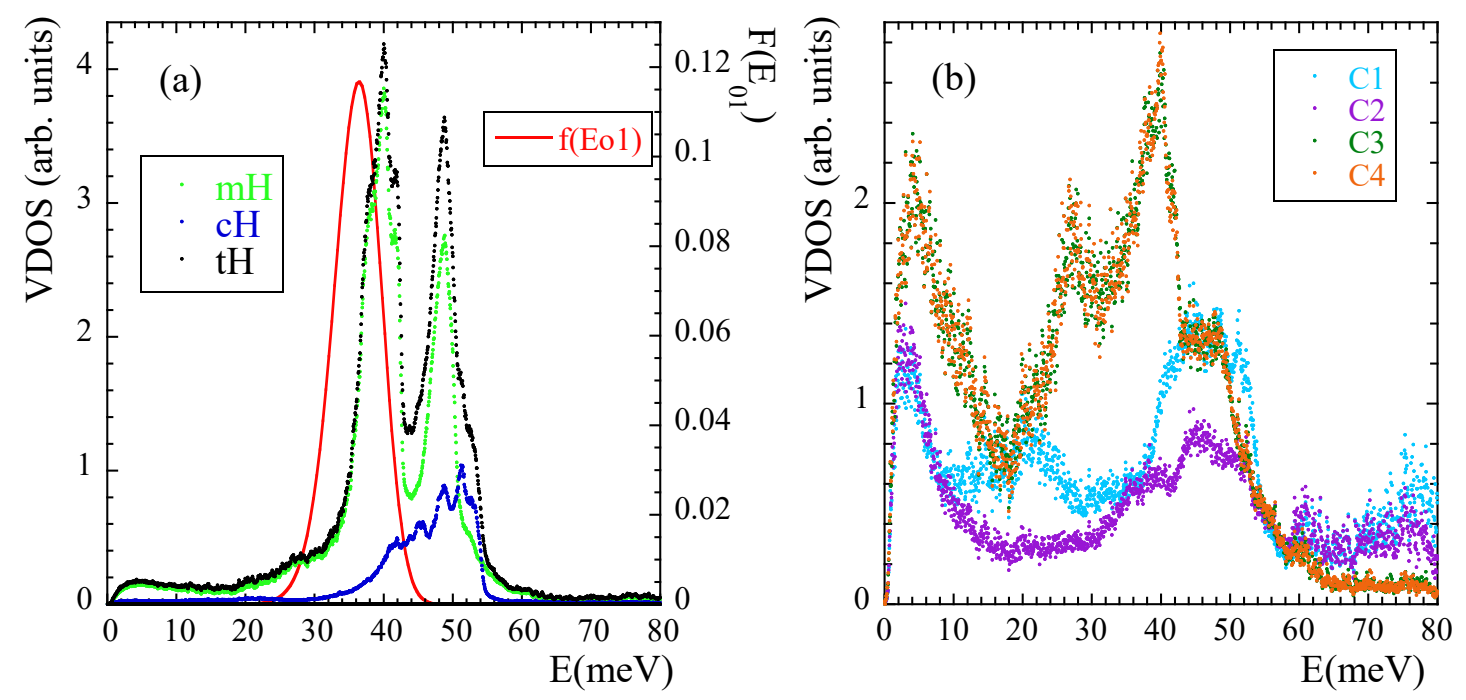

Figure 12. Vibrational density of states calculated at $25 \mathrm{~K}$ for $\mathrm{mH}, \mathrm{cH}$ and $\mathrm{tH}$ (a) and for the different carbons [C1: methylene cC; $\mathrm{C} 2$ : quaternary $\mathrm{cC} ; \mathrm{C} 3$ and $\mathrm{C} 4: \mathrm{mC}](\mathbf{b})$. The line in (a) represents the librational peak $F\left(E_{01}\right)$ deduced within the 3-fold approximation from the distribution of activation energies obtained from the analysis of the functions $F_{s}^{r e l}(Q, t)$ at high temperatures.

\subsection{Revisiting Neutron Scattering Studies}

As it was mentioned in the Introduction, to shed light on the origin of the dielectric $\beta$ process, in Ref. [34] backscattering NS-measurements were performed on fully protonated PIB in the temperature range $260-280 \mathrm{~K}$. There, the $\alpha$-relaxation was not expected to sensitively contribute in the experimental window accessed. Even so, its contribution was considered in the data analysis assuming the merging of $\alpha$ and $\beta$-relaxations as statistically independent processes (i.e., the corresponding intermediate scattering functions are combined as a simple product). The $\beta$-process was represented by a localized motion involving atomic jumps between two equivalent positions separated by a distance $d$. The corresponding EISF is then given by

$$
E I S F=\frac{1}{2}\left(1+\frac{\sin (Q d)}{Q d}\right) .
$$

The resulting value obtained for $d$ was $d=2.7 \AA$. If protonic jumps over such large distances actually occurred in PIB, we could have a chance to identify them in the radial probability distribution function. The best conditions would be at the lowest temperature investigated $(320 \mathrm{~K})$, where we could expect to have the least contribution from the diffusional motions involved in the $\alpha$-process. According to the dielectric results, at $320 \mathrm{~K}$ the occurrence of jumps should manifest at most at about $1 \mathrm{~ns}$ (where the distribution function of the corresponding characteristic times reaches its maximum). As can be seen in the insert of Figure 6a, at such time the function $4 \pi r^{2} G_{s}^{t H}(r, t)$ seems indeed to show a kind of double-peak structure, but the location of the peak (nearly a hump, see arrow in the figure) centered at larger distances would be around, at most, $2 \AA$. In fact, the inspection of this function for $\mathrm{mH}$ and $\mathrm{cH}$ separately reveals that the $\mathrm{cH}$ atoms do not show any clear hint of jumps. Conversely, we have successfully characterized the localized motions of $\mathrm{mH}$ as methyl-group rotations. These are the events producing the second feature at $\approx 2 \AA$ in the distribution function of $\mathrm{tH}$.

It is worth or remark that the result of an apparently large motional amplitude for localized motions arises as a consequence of the deconvolution procedure assuming statistically independence of localized and diffusive-like processes. To illustrate this, we may define an 'apparent EISF' from the values of the above defined deconvoluted function at $t \approx 2$ ns. At this time, $F_{s}^{\text {Deconv }}(Q, t)$ seems to approach a plateau at low tempera- 
tures, as shown in Figure 7a. To take into account the different vibrational dynamics of $\mathrm{mH}$ and $\mathrm{cH}$, we have corrected the results by the value of this function at $t=2 \mathrm{ps}$, $E_{S S F_{a p p}} \equiv F_{s}^{\text {Deconv }}(Q, t=2 n s) / F_{s}^{\text {Deconv }}(Q, t=2 p s)$. The resulting values are shown as circles in Figure 8a. This kind of data treatment for $\mathrm{mH}$ motions results in apparent EISF-values that are lower than those corresponding to a methyl-group rotation. The fit of Equation (18) to the such obtained data in the experimentally accessed $Q$-window deliver a jump distance of $2.6 \AA$ (see solid line in Figure 8a). Thus, the coupling between methyl group rotations and main-chain dynamics in PIB invalidates for this polymer the hypothesis of diffusive and localized motions being statistically independent processes.

\section{Conclusions}

In this work we have shown the power of properly validated fully atomistic molecular dynamics simulations to unravel the details of the atomic motions at inter- and intramolecular length scales in polymers. First of all, the realism of the simulated cell has been proved by direct comparison of magnitudes calculated from the simulated atomic trajectories and determined on the real polymer by neutron scattering experiments. Taking advantage of the information available from the atomic trajectories, the simulations have been exploited to disentangle the self-motions of the different kinds of nuclei in PIB. The different atomic species display distinct motions, being rotations of methyl groups coupled with main-chain dynamics; in all cases, a crossover from Gaussian to non-Gaussian behavior is observed. In more detail, we have shown that:

- The motions of the different atomic species are distinct. All atoms manifest Gaussianlike behavior at low $Q$-values and deviations from it at high $Q$ s.

- The anomalous jump diffusion model captures this behavior by invoking an underlying distribution of elementary jumps at the origin of the sublinear diffusion of atoms in the $\alpha$-relaxation regime. The typical lengths of the jumps deduced are $\approx 0.4 \AA$ for main-chain atoms, in accordance with results reported for other polymers.

- Due to rotations, methyl-group hydrogens display an enhanced mobility in the region where the other atoms show decaging processes. These motions are manifested in low values of the shape parameter and shorter characteristic times at small length scales.

- The usually invoked statistically independence of rotation and translational motions does not work in polyisobutylene. The methyl-group rotations could be fully characterized by analyzing the relative motion of these atoms with respect to the carbon at which the are linked. The rotational rate distribution model gives account for these motions, though deviations are found at high $Q$-values. They can be rationalized as due to non-Gaussian processes taking place during the cageing regime of backbone atoms, which allow a superimposed motion of the rotation axis.

- The methyl-group rotations are coupled with the main-chain dynamics, as deduced from the coincidence of the characteristic times for rotations and non-Gaussian events within the cage of main-chain carbons. This coupling was also concluded from a study combining MD-simulations and NMR experiments [38] and has also been deduced from the analysis of the coherent structure factor of the present simulations [55]. This shall be the origin of the high value observed for the average potential barrier for methyl-group dynamics, leading consequently to a high value of the librational frequency. In fact, a double peak appears in the VDOS of methyl-group hydrogens, where the peak at higher energies occurs where main-chain carbons also present a characteristic peak.

- The information provided by the simulations in real space rules out the occurrence of large amplitude jumps $(\approx 3 \AA)$ associated to the $\beta$-process, as proposed from the analysis of neutron scattering data on protonated samples [34]. The deduction of such a motion is an artifact arising when the hypothesis of statistically independence of $\alpha$-relaxation and localized processes is assumed. The reason is the coupling of methylgroup rotations and main-chain dynamics, possibility that was already suggested by 
the authors of Ref. [34] as an explanation of the apparently incompatible observations from incoherent and coherent scattering studies.

Supplementary Materials: The following are available online at www.mdpi.com/xxx/s1.

Author Contributions: Conceptualization, J.C.; methodology, J.C.; software, F.A.; validation, Y.K., J.C. F.A. and A.A.; data treatment and analysis, Y.C., J.C., A.A. and F.A.; Writing-Original draft preparation, A.A.; Writing-Review and editing, J.C and A.A.; funding acquisition, J.C. All authors have read and agreed to the published version of the manuscript.

Funding: This research was funded by the Basque Government, code: IT-1175-19 and the Ministerio de Economía y Competitividad code: PGC2018-094548-B-I00 (MCIU / AEI/FEDER, UE).

Institutional Review Board Statement: Not applicable.

Informed Consent Statement: Not applicable.

Data Availability Statement: The data presented in this study are openly available in: https:// ehubox.ehu.eus/s/6zpwz274ntyw5tt.

Acknowledgments: We thank all our collaborators in the neutron scattering facilities all over the world who, along the past decades, have participated in the experiments. We also would like to thank the rest of the members of our research group in San Sebastián as well as Prof. Dieter Richter and his group in Jülich for the long-standing and fruitful collaboration.

Conflicts of Interest: The authors declare no conflict of interest.

\section{Abbreviations}

The following abbreviations are used in this manuscript:

$\begin{array}{ll}\text { NS } & \text { Neutron scattering } \\ \text { PVME } & \text { poly(vinyl methyl ether) } \\ \text { PH } & \text { Phenoxy } \\ \text { PVC } & \text { Poly(vinyl chloride) } \\ \text { PB } & \text { 1,4-Polybutadiene } \\ \text { PVE } & \text { Poly(vinyl ethylene) } \\ \text { PI } & \text { Polyisoprene } \\ \text { PIB } & \text { Polyisobutylene } \\ \text { NSE } & \text { Neutron spin echo } \\ \text { MD } & \text { Molecular dynamics } \\ \text { PMMA } & \text { Poly(methyl metacrylate) } \\ \text { PEP } & \text { Poly(ethylene propylene) } \\ \text { hh-PP } & \text { head-to-head Polypropylene } \\ \text { PVAc } & \text { Poly(vinyl acetate) } \\ \text { PVP } & \text { Poly(vinylpyrrolidone) } \\ \text { PEMA } & \text { Poly(ethyl methacrylate) } \\ \text { PTHF } & \text { Poly(tetrahydrofurane) } \\ \text { VDOS } & \text { vibrational density of states } \\ \text { cC } & \text { main-chain carbons } \\ \text { cH } & \text { main-chain hydrogens } \\ \text { mC } & \text { methyl-group carbons } \\ \text { mH } & \text { methyl-group hydrogens } \\ \text { tH } & \text { total hydrogens } \\ \text { SBR } & \text { styrene-butadiene-rubber } \\ \text { PS } & \text { polystyrene } \\ & \end{array}$

\section{References}

1. Richter, D.; Monkenbusch, M.; Arbe, A.; Colmenero, J. Neutron Spin Echo in Polymer Systems; Springer: Berlin/Heidelberg, Germany; New York, NY, USA, 2005; Volume 174.

2. Colmenero, J.; Arbe, A. Recent progress on polymer dynamics by neutron scattering: From simple polymers to complex materials. J. Polym. Sci. Part B Polym. Phys. 2013, 51, 87-113. [CrossRef] 
3. Arbe, A.; Alvarez, F.; Colmenero, J. Neutron scattering and molecular dynamics simulations: Synergetic tools to unravel structure and dynamics in polymers. Soft Matter 2012, 8, 8257-8270. [CrossRef]

4. Colmenero, J.; Alegría, A.; Arbe, A.; Frick, B. Correlation between non-Debye behavior and $Q$ behavior of the $\alpha$ relaxation in glass-forming polymeric systems. Phys. Rev. Lett. 1992, 69, 478-481. [CrossRef]

5. Arbe, A.; Colmenero, J.; Monkenbusch, M.; Richter, D. Dynamics of Glass-Forming Polymers: 'Homogeneous' versus 'Heterogeneous' Scenario. Phys. Rev. Lett. 1998, 81, 590-593. [CrossRef]

6. Triolo, A.; Russina, O.; Arrighi, V.; Juranyi, F.; Janssen, S.; Gordon, C.M. Quasielastic neutron scattering characterization of the relaxation processes in a room temperature ionic liquid. J. Chem. Phys. 2003, 119, 8549-8557. [CrossRef]

7. Kofu, M.; Faraone, A.; Tyagi, M.; Nagao, M.; Yamamuro, O. Two inherent crossovers of the diffusion process in glass-forming liquids. Phys. Rev. E 2018, 98, 042601. [CrossRef]

8. Sobolev, O.; Novikov, A.; Pieper, J. Quasielastic neutron scattering and microscopic dynamics of liquid ethylene glycol. Chem. Phys. 2007, 334, 36-44. [CrossRef]

9. Roh, J.H.; Tyagi, M.; Hogan, T.E.; Roland, C.M. Space-dependent dynamics in 1,4-polybutadiene nanocomposite. Macromolecules 2013, 46, 6667-6669. [CrossRef]

10. Jhalaria, M.; Buenning, E.; Huang, Y.; Tyagi, M.; Zorn, R.; Zamponi, M.; García-Sakai, V.; Jestin, J.; Benicewicz, B.C.; Kumar, S.K. Accelerated local dynamics in matrix-free polymer grafted nanoparticles. Phys. Rev. Lett. 2019, 123, 158003. [CrossRef]

11. Toppozini, L.; Roosen-Runge, F.; Bewley, R.I.; M. Dalgliesh, R.; Perring, T.; Seydel, T.; Glyde, H.R.; García Sakai, V.; Rheinstädter, M.C. Anomalous and anisotropic nanoscale diffusion of hydration water molecules in fluid lipid membranes. Soft Matter 2015, 11, 8354-8371. [CrossRef]

12. Vural, D.; Smith, J.C.; Petridis, L. Dynamics of the lignin glass transition. Phys. Chem. Chem. Phys. 2018, 20, 20504-20512. [CrossRef]

13. Farago, B.; Arbe, A.; Colmenero, J.; Faust, R.; Buchenau, U.; Richter, D. Intermediate length scale dynamics of polyisobutylene. Phys. Rev. E 2002, 65, 051803. [CrossRef]

14. Arbe, A.; Colmenero, J.; Alvarez, F.; Monkenbusch, M.; Richter, D.; Farago, B.; Frick, B. Non-Gaussian nature of the $\alpha$ relaxation of glass-forming polyisoprene. Phys. Rev. Lett. 2002, 89, 245701. [CrossRef]

15. Arbe, A.; Colmenero, J.; Alvarez, F.; Monkenbusch, M.; Richter, D.; Farago, B.; Frick, B. Experimental evidence by neutron scattering of a crossover from Gaussian to non-Gaussian behavior in the $\alpha$ relaxation of polyisoprene. Phys. Rev. E 2003, 67, 051802. [CrossRef]

16. Arbe, A.; Alvarez, F.; Colmenero, J. Insight into the structure and dynamics of polymers by neutron scattering combined with atomistic molecular dynamics simulations. Polymers 2020, 12, 3067. [CrossRef]

17. Singwi, K.S.; Sjölander, A. Diffusive motions in water and cold neutron scattering. Phys. Rev. 1960, 119, 863-871. [CrossRef]

18. Colmenero, J.; Arbe, A.; Alvarez, F.; Narros, A.; Richter, D. Hydrogen motions and the $\alpha$-relaxation in glass- forming polymers: Molecular dynamics simulation and quasi-elastic neutron scattering results. Pramana J. Phys. 2004, 63, 25-32. [CrossRef]

19. Narros, A.; Alvarez, F.; Arbe, A.; Colmenero, J.; Richter, D.; Farago, B. Hydrogen motions in the $\alpha$-relaxation regime of poly (vinyl ethylene): A molecular dynamics simulation and neutron scattering study. J. Chem. Phys. 2004, 121, 3282. [CrossRef]

20. Capponi, S.; Arbe, A.; Cerveny, S.; Busselez, R.; Frick, B.; Embs, J.; Colmenero, J. Quasielastic neutron scattering study of hydrogen motions in an aqueous poly(vinyl methyl ether) solution. J. Chem. Phys. 2011, 134, 204906. [CrossRef]

21. Genix, A.C.; Arbe, A.; Alvarez, F.; Colmenero, J.; Farago, B.; Wischnewski, A.; Richter, D. Self- and collective dynamics of syndiotactic poly(methyl methacrylate). A combined study by quasielastic neutron scattering and atomistic molecular dynamics simulations. Macromolecules 2006, 39, 6260-6272. [CrossRef]

22. Aparicio, R.P.; Arbe, A.; Colmenero, J.; Frick, B.; Willner, L.; Richter, D. Quasielastic neutron scattering study on the effect of blending on the dynamics of head-to-head poly(propylene) and poly(ethylene-propylene). Macromolecules 2006, 39, 1060-1072. [CrossRef]

23. Tyagi, M.; Arbe, A.; Alegría, A.; Colmenero, J.; Frick, B. Dynamic confinement effects in polymer blends. A quasielastic neutron scattering study of the slow component in the blend poly (vinyl acetate)/poly (ethylene oxide). Macromolecules 2007, 40, 4568. [CrossRef]

24. Busselez, R.; Arbe, A.; Alvarez, F.; Colmenero, J.; Frick, B. Study of the structure and dynamics of poly(vinyl pyrrolidone) by molecular dynamics simulations validated by quasielastic neutron scattering and X-ray diffraction experiments. J. Chem. Phys. 2011, 134, 054904. [CrossRef] [PubMed]

25. Genix, A.C.; Arbe, A.; Colmenero, J.; Wuttke, J.; Richter, D. Neutron scattering and X-ray investigation of the structure and dynamics of poly(ethyl methacrylate). Macromolecules 2012, 45, 2522. [CrossRef]

26. Arbe, A.; Rubio, J.; Malo de Molina, P.; Maiz, J.; Pomposo, J.A.; Fouquet, P.; Prevost, S.; Juranyi, F.; Khaneft, M.; Colmenero, J. Melts of single-chain nanoparticles: A neutron scattering investigation. J. Appl. Phys. 2020, 127, 044305. [CrossRef]

27. Arrighi, V.; Tanchawanich, J.; Telling, M.T.F. Molar mass dependence of polyethylene chain dynamics. A quasi-elastic neutron scattering investigation. Macromolecules 2013, 46, 216-225. [CrossRef]

28. Busselez, R.; Lefort, R.; Ghoufi, A.; Beuneu, B.; Frick, B.; Affouard, F.; Morineau, D. The non-Gaussian dynamics of glycerol. J. Phys. Condens. Matter 2011, 23, 505102. [CrossRef] 
29. Perticaroli, S.; Mostofian, B.; Ehlers, G.; Neuefeind, J.C.; Diallo, S.O.; Stanley, C.B.; Daemen, L.; Egami, T.; Katsaras, J.; Cheng, X.; et al. Structural relaxation, viscosity, and network connectivity in a hydrogen bonding liquid. Phys. Chem. Chem. Phys. 2017, 19, 25859-25869. [CrossRef]

30. Busselez, R.; Lefort, R.; Guendouz, M.; Frick, B.; Merdrignac-Conanec, O.; Morineau, D. Molecular dynamics of glycerol and glycerol-trehalose bioprotectant solutions nanoconfined in porous silicon. J. Chem. Phys. 2009, 130, 214502. [CrossRef]

31. Arbe, A.; Buchenau, U.; Willner, L.; Richter, D.; Farago, B.; Colmenero, J. Study of the dynamic structure factor in the $\beta$-relaxation regime of polybutadiene. Phys. Rev. Lett. 1996, 76, 1872. [CrossRef] [PubMed]

32. Arbe, A.; Richter, D.; Colmenero, J.; Farago, B. Merging of the $\alpha$ and $\beta$ relaxations in polybutadiene: A neutron spin echo and dielectric study. Phys. Rev. E 1996, 54, 3853. [CrossRef] [PubMed]

33. Narros, A.; Arbe, A.; Alvarez, A.; Colmenero, J.; Richter, D. Atomic motions in the $\alpha \beta$-merging region of 1,4-polybutadiene: A molecular dynamics simulation study. J. Chem. Phys. 2008, 128, 224905. [CrossRef]

34. Arbe, A.; Colmenero, J.; Frick, B.; Monkenbusch, M.; Richter, D. Investigation of the dielectric $\beta$-process in polyisobutylene by incoherent quasielastic neutron scattering. Macromolecules 1998, 31, 4926-4934. [CrossRef] [PubMed]

35. Richter, D.; Arbe, A.; Colmenero, J.; Monkenbusch, M.; Farago, B.; Faust, R. Molecular motions in polyisobutylene: A neutron spin echo and dielectric investigation. Macromolecules 1998, 31, 1133-1143. [CrossRef]

36. Slichter, W.P. NMR studies of multiple relaxations in polymers. J. Polym. Sci. Part C 1966, 14, 33. [CrossRef]

37. Törmälä, P. Spin label and probe studies of polymeric solids and melts. J. Macromol. Sci. Rev. Macromol. Chem. C 1979, 17, 297. [CrossRef]

38. Karatasos, K.; Ryckaert, J.P.; Ricciardi, R.; Laupretre, F. Methyl dynamics and $\beta$-relaxation in polyisobutylene: Comparison between experiment and molecular dynamics simulations. Macromolecules 2002, 35, 1451-1462. [CrossRef]

39. Frick, B.; Richter, D.; Trevino, S. Inelastic fast relaxation in a weakly fragile polymer glass near Tg. Phys. A 1993, 201, 88-94. [CrossRef]

40. Frick, B.; Richter, D. Change of the vibrational dynamics near the glass transition in polyisobutylene: Inelastic neutron scattering on a nonfragile polymer. Phys. Rev. B 1993, 47, 14795. [CrossRef]

41. Colmenero, J.; Moreno, A.J.; Alegría, A. Neutron scattering investigations on methyl group dynamics in polymers. Prog. Polym. Sci. 2005, 30, 1147-1184. [CrossRef]

42. Adams, M.A.; Gabrys, B.J.; Zajac, W.M.; Peiffer, D.G. High-resolution incoherent inelastic neutron scattering spectra of polyisobutylene and polyisoprene. Macromolecules 2005, 38, 160-166. [CrossRef]

43. Londono, J.D.; Habenschuss, A.; Curro, J.G.; Rajasekaran, J.J. Short-range order in some polymer melts from X-ray diffraction. J. Polym. Sci. Part B Polym. Phys. 1996, 34, 3055-3061. [CrossRef]

44. Richter, D.; Monkenbusch, M.; Allgeier, J.; Arbe, A.; Colmenero, J.; Farago, B.; Cheol Bae, Y.; Faust, R. From Rouse dynamics to local relaxation: A neutron spin echo study on polyisobutylene melts. J. Chem. Phys. 1999, 111, 6107-6120. [CrossRef]

45. Kanaya, T.; Kawaguchi, T.; Kaji, K. Local dynamics of some bulk polymers above $\mathrm{T}_{g}$ as seen by quasielastic neutron scattering. Macromolecules 1999, 32, 1672-1678. [CrossRef]

46. Kisliuk, A.; Mathers, R.T.; Sokolov, A.P. Crossover in dynamics of polymeric liquids: Back to T $\ell_{\ell}$ ? J. Polym. Sci. Part B Polym. Phys. 2000, 38, 2785. [CrossRef]

47. Triolo, A.; Lechner, R.E.; Desmedt, A.; Telling, M.T.F.; Arrighi, V. Complex dynamics in polyisobutylene melts. Macromolecules 2002, 35, 7039-7043. [CrossRef]

48. Arrighi, V.; Triolo, A.; Qian, H. Temperature dependence of the segmental dynamics in polyisobutylene melts. J. Non-Cryst. Solids 2002, 307-310, 654-657. [CrossRef]

49. Ganazzoli, F.; Raffaini, G.; Arrighi, V. The stretched-exponential approximation to the dynamic structure factor in non-entangled polymer melts. Phys. Chem. Chem. Phys. 2002, 4, 3734-3742. [CrossRef]

50. Ding, Y.; Novikov, V.N.; Sokolov, A.P.; Dalle-Ferrier, C.; Alba-Simionesco, C.; Frick, B. Influence of molecular weight on fast dynamics and fragility of polymers. Macromolecules 2004, 37, 9264-9272. [CrossRef]

51. Begen, B.; Kisliuk, A.; Novikov, V.; Sokolov, A.; Niss, K.; Chauty-Cailliaux, A.; Alba-Simionesco, C.; Frick, B. Influence of pressure on fast dynamics in polyisobutylene. J. Non-Cryst. Solids 2006, 42-49, 4583-4588. [CrossRef]

52. Niss, K.; Begen, B.; Frick, B.; Ollivier, J.; Beraud, A.; Sokolov, A.; Novikov, V.N.; Alba-Simionesco, C. Influence of Pressure on the Boson Peak: Stronger than Elastic Medium Transformation. Phys. Rev. Lett. 2007, 99, 055502. [CrossRef] [PubMed]

53. Niss, K.; Dalle-Ferrier, C.; Giordano, V.M.; Monaco, G.; Frick, B.; Alba-Simionesco, C. Glassy properties and viscous slowing down: An analysis of the correlation between nonergodicity factor and fragility. J. Chem. Phys. 2008, 129, 194513. [CrossRef] [PubMed]

54. Dalle-Ferrier, C.; Niss, K.; Sokolov, A.P.; Frick, B.; Serrano, J.; Alba-Simionesco, C. The role of chain length in nonergodicity factor and fragility of polymers. Macromolecules 2010, 43, 8977-8984. [CrossRef]

55. Khairy, Y.; Alvarez, F.; Arbe, A.; Colmenero, J. Collective features in polyisobutylene. A study of the static and dynamic structure factor by molecular dynamics simulations. Macromolecules 2014, 47, 447. [CrossRef]

56. Tengroth, C.; Engberg, D.; Wahnström, G.; Börjesson, L.; Carlsson, P.; Ahlström, P.; Howells, W.S. The segmental and rotational dynamics of PPO, above the glass-transition, investigated by neutron scattering and molecular dynamics simulations. Soft Mater. 2005, 3, 1-20. [CrossRef] 
57. Zhang, C.; Arrighi, V.; Gagliardi, S.; McEwen, I.J.; Tanchawanich, J.; Telling, M.T.; Zanotti, J.M. Quasielastic neutron scattering measurements of fast process and methyl group dynamics in glassy poly(vinyl acetate). Chem. Phys. 2006, 328, 53-63. [CrossRef]

58. Schönhals, A.; Goering, H.; Schick, C.; Frick, B.; Zorn, R. Glassy dynamics of polymers confined to nanoporous glasses revealed by relaxational and scattering experiments. Eur. Phys. J. E 2003, 12, 173-178. [CrossRef]

59. Arrighi, V.; Gagliardi, S.; Zhang, C.; Ganazzoli, F.; Higgins, J.S.; Ocone, R.; Telling, M.T.F. A unified picture of the local dynamics of poly(dimethylsiloxane) across the melting point. Macromolecules 2003, 36, 8738-8748. [CrossRef]

60. Chen, C.; Maranas, J.K.; García-Sakai, V. Local dynamics of syndiotactic poly(methyl methacrylate) using molecular dynamics simulation. Macromolecules 2006, 39, 9630-9640. [CrossRef]

61. Zorn, R.; Frick, B.; Fetters, L.J. Quasielastic neutron scattering study of the methyl group dynamics in polyisoprene. J. Chem. Phys. 2002, 116, 845-853. [CrossRef]

62. Smuda, C.; Busch, S.; Wagner, B.; Unruh, T. Methyl group dynamics in glassy, polycrystalline, and liquid coenzyme Q10 studied by quasielastic neutron scattering. J. Chem. Phys. 2008, 129, 074507. [CrossRef]

63. Fetters, L.J.; Lohse, D.J.; Richter, D.; Witten, T.A.; Zirkel, A. Connection between polymer molecular weight, density, chain dimensions, and melt viscoelastic properties. Macromolecules 1994, 27, 4639. [CrossRef]

64. Springer, T. Quasielastic Neutron Scattering for the Investigation of Diffusive Motions in Solids and Liquids; Springer Tracts in Modern Physics; Springer: Berlin/Heidelberg, Germany; New York, NY, USA, 1972; Volume 64.

65. Squires, G.L. Introduction to the Theory of Thermal Neutron Scattering; Dover Publication Inc.: New York, NY, USA, 1996.

66. Zorn, R. Deviation from Gaussian behavior in the self-correlation function of the proton motion in polybutadiene. Phys. Rev. B 1997, 55, 6249. [CrossRef]

67. Rahman, A.; Singwi, K.S.; Sjölander, A. Theory of slow neutron scattering by liquids. I. Phys. Rev. 1962, 126, 986. [CrossRef]

68. Mezei, F. Neutron Spin Echo; Lecture Notes in Physics; Springer: Heidelberg, Germany, 1980; Volume 28.

69. Farago, B. IN11C, medium-resolution multidetector extension of the IN11 NSE spectrometer at the ILL. Phys. B 1997, 241-243, 113. [CrossRef]

70. Gambino, T.; Alegría, A.; Arbe, A.; Colmenero, J.; Malicki, N.; Dronet, S.; Schnell, B.; Lohstroh, W.; Nemkovski, K. Applying polymer blend dynamics concepts to a simplified industrial system. A combined effort by dielectric spectroscopy and neutron scattering. Macromolecules 2018, 51, 6692-6706. [CrossRef]

71. Lefort, R.; Morineau, D.; Guégan, R.; Guendouz, M.; Zanotti, J.M.; Frick, B. Relation between static short-range order and dynamic heterogeneities in a nanoconfined liquid crystal. Phys. Rev. E 2008, 78, 040701. [CrossRef]

72. Hopfenmüller, B.; Zorn, R.; Holderer, O.; Ivanova, O.; Lehnert, W.; Lüke, W.; Ehlers, G.; Jalarvo, N.; Schneider, G.J.; Monkenbusch, M.; et al. Fractal diffusion in high temperature polymer electrolyte fuel cell membranes. J. Chem. Phys. 2018, 148, 204906. [CrossRef] [PubMed]

73. Götze, W. Liquids, Freezing, Glass Transition; North-Holland: Amsterdam, The Netherlands, 1991; p. 287.

74. Götze, W. Complex Dynamics of Glass-Forming Liquids. A Mode Coupling Theory; Oxford University Press: New York, NY, USA, 2009.

75. Khairy, Y.; Alvarez, F.; Arbe, A.; Colmenero, J. Applicability of mode-coupling theory to polyisobutylene: A molecular dynamics simulation study. Phys. Rev. E 2013, 88, 042302. [CrossRef]

76. Alvarez, F.; Arbe, A.; Colmenero, J. Methyl group dynamics above the glass transition temperature: A molecular dynamics simulation in polyisoprene. Chem. Phys. 2000, 261, 47. [CrossRef]

77. Chahid, A.; Alegría, A.; Colmenero, J. Methyl group dynamics in poly(vinyl methyl ether). A rotation rate distribution model. Macromolecules 1994, 27, 3282-3288. [CrossRef]

78. Colmenero, J.; Mukhopadhyay, R.; Alegría, A.; Frick, B. Quantum rotational tunneling of methyl groups in polymers. Phys. Rev. Lett. 1998, 80, 2350-2353. [CrossRef] 